\title{
Characterization of Nestin-positive stem Leydig cells as a potential source for the treatment of testicular Leydig cell dysfunction
}

Mei Hua Jiang ${ }^{1,2,3, *}$, Bing Cai ${ }^{2,4,}$, Ying Tuo ${ }^{2,5, *}$, Jiancheng Wang ${ }^{2}$, Zhi Jun Zang ${ }^{6}$, Xiang'an Tu ${ }^{7}$, Yong Gao ${ }^{4}$, Zhijian $\mathrm{Su}^{8}$, Weiqiang $\mathrm{Li}^{2,9}$, Guilan $\mathrm{Li}^{2}$ Min Zhang ${ }^{2}$, Jianwei Jiao ${ }^{10}$, Zi Wan ${ }^{7}$, Chunhua Deng ${ }^{7}$, Bruce $\mathrm{T} \mathrm{Lahn}^{2}$, Andy Peng Xiang 1, 2,3,4,9

${ }^{I}$ Cell-gene Therapy Translational Medicine Research Center, The Third Affiliated Hospital, Zhongshan School of Medicine, Sun Yat-sen University, Guangzhou, Guangdong 510630, China; ${ }^{2}$ Key Laboratory for Stem Cells and Tissue Engineering, Center for Stem Cell Biology and Tissue Engineering, Ministry of Education, ${ }^{3}$ Department of Anatomy, Zhongshan School of Medicine, Sun Yat-sen University, Guangzhou, Guangdong 510080, China; ${ }^{4}$ Reproductive Medicine Center and Guangdong provincial Key Laborartory of Reproductive Medicine, ${ }^{5}$ Department of Histopathology, The First Affiliated Hospital, Sun Yat-sen University, Guangzhou, Guangdong 510080, China; ${ }^{6}$ Department of Infertility and Sexual Medicine, The Third Affiliated Hospital, Sun Yat-sen University, Guangzhou, Guangdong 510630, China; ${ }^{7}$ Department of Urology, The First Affiliated Hospital, Sun Yat-Sen University, Guangzhou, Guangdong 510080, China; ${ }^{8}$ Key Laboratory of Bioengineering Medicine of Guangdong Province, Institute of Biomedicine, Jinan University, Guangzhou, Guangdong 510632, China; ${ }^{9}$ Department of Biochemistry, Zhongshan School of Medicine, Sun Yat-sen University, Guangzhou, Guangdong 510080, China, ${ }^{10}$ State key Laboratory of Reproductive Biology, Institute of Zoology, Chinese Academy of Sciences, Beijing 100101, China

The ability to identify and isolate lineage-specific stem cells from adult tissues could facilitate cell replacement therapy. Leydig cells (LCs) are the primary source of androgen in the mammalian testis, and the prospective identification of stem Leydig cells (SLCs) may offer new opportunities for treating testosterone deficiency. Here, in a transgenic mouse model expressing GFP driven by the Nestin (Nes) promoter, we observed Nes-GFP ${ }^{+}$cells located in the testicular interstitial compartment where SLCs normally reside. We showed that these Nes-GFP ${ }^{+}$cells expressed LIFR and PDGFR-a, but not LC lineage markers. We further observed that these cells were capable of clonogenic self-renewal and extensive proliferation in vitro and could differentiate into neural or mesenchymal cell lineages, as well as LCs, with the ability to produce testosterone, under defined conditions. Moreover, when transplanted into the testes of LC-disrupted or aging models, the Nes-GFP ${ }^{+}$cells colonized the interstitium and partially increased testosterone production, and then accelerated meiotic and post-meiotic germ cell recovery. In addition, we further demonstrated that CD51 might be a putative cell surface marker for SLCs, similar with Nestin. Taken together, these results suggest that Nes-GFP ${ }^{+}$cells from the testis have the characteristics of SLCs, and our study would shed new light on developing stem cell replacement therapy for testosterone deficiency.

Keywords: stem Leydig cell; Nestin; self-renewal; multipotency; Leydig cell dysfunction

Cell Research (2014) 24:1466-1485. doi:10.1038/cr.2014.149; published online 21 November 2014

\section{Introduction}

*These three authors contributed equally to this work.

Correspondence: Andy Peng Xiang

Tel: +86-20-87335822; Fax: +86-20-87335858

E-mail: xiangp@mail.sysu.edu.cn

Received 14 September 2013; revised 19 January 2014; accepted 1 September 2014; published online 21 November 2014
Leydig cells (LCs), which reside in the testis interstitium, are the primary source of testosterone and essential for the development of the male phenotype and spermatogenesis $[1,2]$. Testosterone is also important for the maintenance of bone and muscle mass. A recent epidemiological study estimated that more than 4.5 million American men may have symptoms related to testosterone deficiency, such as changes in body composition, 
increased fatigue, sexual dysfunction, depressed mood, and decreased cognitive function [3, 4]. Although LC transplantation has been demonstrated as an ideal physiological and long-acting system for testosterone delivery [5], adult LCs have a limited capacity to proliferate, and display all of the phenotypic and functional features of terminally differentiated cells $[6,7]$, thereby limiting the efficacy of LC transplantation therapy. Thus, identification and isolation of stem Leydig cells (SLCs), which are capable of self-renewal and differentiating into mature LCs, will provide a new strategy for treating the testosterone deficiency.

Recently, several putative SLC candidates have been described in the testis. Lo et al. [8] firstly reported the isolation of putative SLCs from the testicular side population (SP), and transplantation of this population could increase testosterone production and restore spermatogenesis in luteinizing hormone receptor (LHR) knockout (KO) mice. Unfortunately, the testicular SP cells were heterogeneous and contained different types of stem cells, including spermatogonial stem cells (SSCs), LCs, and perhaps myoid stem cells. Therefore, this population might not be the best choice for the isolation of SLCs. Hardy and his colleagues made significant progress in search for SLCs [9]. Through Percoll gradient centrifugation and immunoselection for LHR-negative and platelet-derived growth factor receptor- $\alpha$ (PDGFR- $\alpha$ )-positive cells from day 7 rat testes, putative SLCs were isolated, which were capable of proliferation and differentiation into testosterone-producing LCs in vitro and colonized the interstitium in vivo. Chen's group also demonstrated the existence of SLCs in the adult rat testis [10]. Unfortunately, neither of these studies has completely demonstrated the self-renewal and multilineage-differentiation abilities of putative SLCs, which are the unique properties of adult stem cells, nor did they provide evidence of the potential reparative effects of SLCs on LC functions, particularly the restoration of testosterone production. Furthermore, these isolation methods had a very low efficiency; about one million LHR-negative and PDGFR- $\alpha$-positive cells were obtained from the testes of 60 pups (with an average of 8500 cells per testis) [9]. To overcome the limitations of these methods, particularly the small population size and the heterogeneous nature of the population, there is a clear need for specific markers and methods to identify and prospectively isolate SLCs.

Nestin is an intermediate filament protein. While it is widely known as a marker of neural stem cells, it is actually expressed in proliferating cells during the developmental stages of a variety of embryonic and fetal tissues [11]. More importantly, Nestin is also expressed in some adult stem/progenitor cell populations, indicating that
Nestin might be a common marker of multipotent stem cells. Using Nestin as a tracking marker, various adult stem cells have been prospectively isolated, including mesenchymal stem cells [12] and hair follicle stem cells [13]. Davidoff et al. [14] demonstrated that Nestin expression was elevated in the testis during development and regeneration after LC injury, which implied that Nestin might be a good candidate marker of LC progenitor cells.

Here, we report a method for identification and isolation of SLCs on the basis of Nestin expression using flow cytometry in combination with in vitro functional assays. We further demonstrated that Nestin-positive SLCs had the capacity to partially restore testosterone production in vivo following transplantation into LC-disrupted or senescence animal models.

\section{Results}

\section{Characterization of Nestin expression in the testis}

To systemically evaluate the expression patterns of Nestin during postnatal development in the mouse testes, we assessed GFP expression in the Nes-GFP transgenic mouse model (expressing GFP driven by the Nestin (Nes) promoter) using confocal microscopy. Consistent with previous reports $[14,15]$, we detected GFP expression in the interstitial or peritubular cells of the mouse testes on postnatal days $0,7,14,28$ and 90 , but not within the seminiferous tubules (Figure 1A). Quantitative analysis showed a significant peak of the number of $N e s-\mathrm{GFP}^{+}$ cells on postnatal day 7 , which then gradually decreased with age in the total population of the interstitial compartment; by day 90 , Nes-GFP ${ }^{+}$cells were barely seen in the testis interstitium (Figure 1B). Additionally, using specific Nestin antibodies yielded similar staining patterns in these tissues (data not shown). No staining was found in the negative controls.

To further characterize Nes-GFP ${ }^{+}$cells, we examined the expression of known stem cell or LC lineage markers in $N e s-G F P^{+}$cells. On postnatal day 7, the Nes-GFP cells expressed PDGFR- $\alpha$ and leukemia inhibitory factor receptor (LIFR), but did not express the fetal LC marker LHR or the adult LC marker 3 $\beta$-HSD [9] (Figure 1C). Furthermore, we observed that only a few Nes-GFP ${ }^{+}$cells expressed c-kit (data not shown). We also monitored the mRNA levels of Nestin and genes associated with SLCs in the testis tissue using quantitative RT-PCR (qRT-PCR) analyses. In agreement with previous observations [14], the expression level of Nestin gene was gradually decreased with age (Figure 1D). Interestingly, the expression pattern of $P D G F R-\alpha$ and $L I F R$ genes resembled that of Nestin gene in the testis $[16,17]$ (Figure 1D). Taken 
A
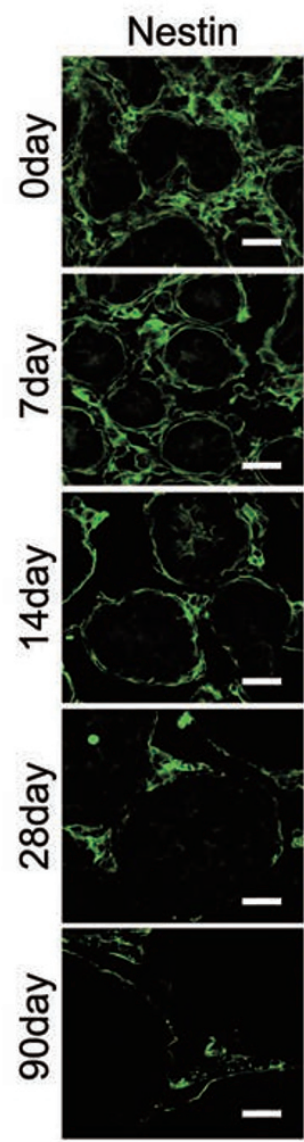

B

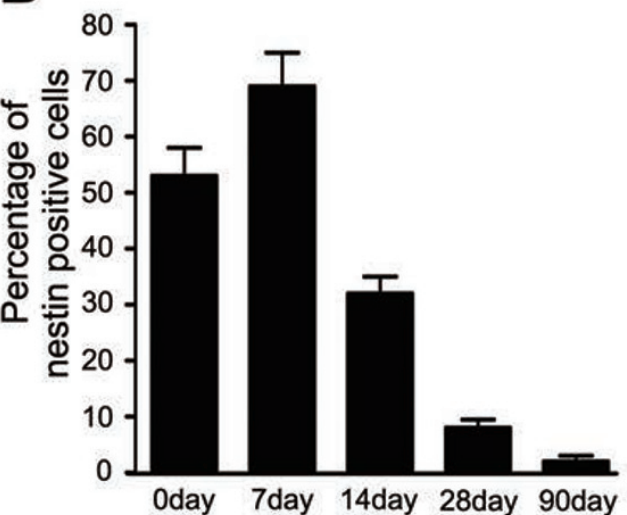

Merge
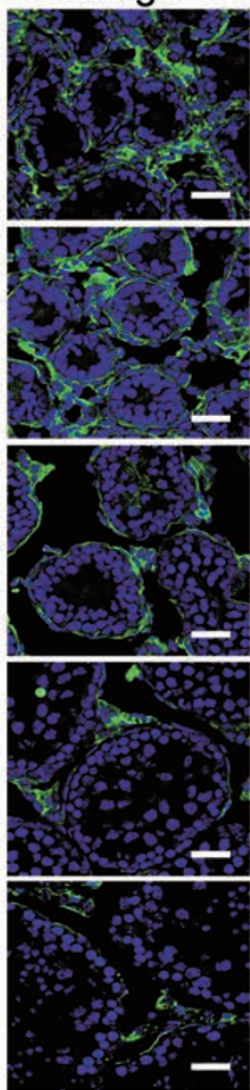

D

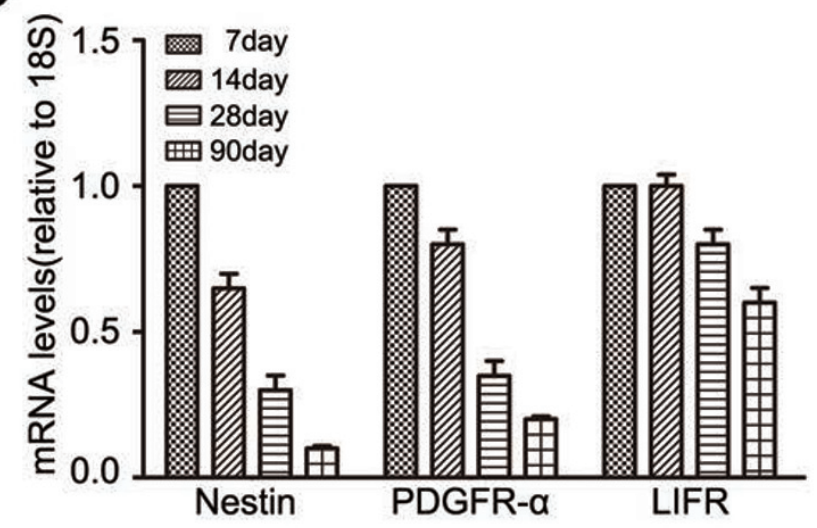

Figure 1 Nestin expression in the testis of Nes-GFP transgenic mice. (A) Nestin (green) expression in the testis of Nes-GFP transgenic mice on postnatal days $0,7,14,28$, and 90 . Scale bar, $50 \mu \mathrm{m}$. (B) Quantification of Nes-GFP ${ }^{+}$cells in the testis sections at the indicated time points. The percentage of Nes-GFP ${ }^{+}$cells in the testis interstitum was counted. Three sections per slide and three slides per mouse testis were counted. (C) Immunohistochemical analyses showed that the Nes-GFP

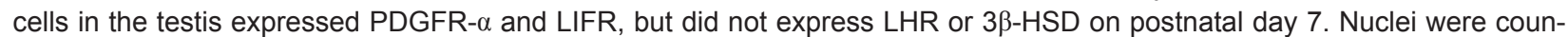
terstained with DAPI (blue). Scale bar, $50 \mu \mathrm{m}$. (D) qRT-PCR analysis of Nestin, PDGFR- $\alpha$ and LIFR gene expression in testes of normal mice on postnatal days $7,14,28,90$ (the value on postnatal day 7 was defined as 1 ). $n=5$. Data are expressed as the mean \pm SEM. 
together, these results suggest that Nestin might be a reliable marker for SLCs.

Isolation of Nes-GFP $\mathrm{Fell}^{+}$from the testis

Histological data demonstrated that Nestin represents a potential marker for SLCs, suggesting the possibility of isolating SLCs on the basis of Nes-GFP expression. Towards this end, we sorted cells from the testes of postnatal Nes-GFP transgenic mice using fluorescence-activated cell sorting (FACS) and found that $N e s-\mathrm{GFP}^{+}$cells constituted $52.1 \% \pm 5.6 \%, 29.5 \% \pm 6.3 \%$, and $1.3 \% \pm$ $0.4 \%$ of the total testicular interstitial cell population on postnatal days 7,14 , and 28 , respectively. Consistent with the histological data the frequency of $\mathrm{GFP}^{+}$cells de- creased with age (Figure 2A).

We then examined the expression of GFP, PDGFR- $\alpha$, and LHR in freshly prepared testicular cells from postnatal day $7 \mathrm{Nes}$-GFP mice. These cells were almost uniformly LHR-negative (Figure 2B), whereas they displayed a more heterogeneous expression of PDGFR- $\alpha$ (Figure 2C). These cells were separated into four distinct fractions: Nes-GFP ${ }^{+}$PDGFR- $\alpha^{+}$(double positive; DP), Nes-GFP ${ }^{+} / \mathrm{PDGFR}-\alpha^{-}$(Nes-GFP ${ }^{+}$single positive; NSP), Nes-GFP ${ }^{-} /$PDGFR- $\alpha^{+}$(PDGFR- $\alpha$ single positive; PSP), and $N e s-\mathrm{GFP}^{-} / \mathrm{PDGFR}-\alpha^{-}$(double negative; DN; Figure $2 \mathrm{C}$ ). As shown in Figure 2C, the proportion of each fraction was $37.0 \%$ (DP), $18.4 \%$ (NSP), $12.1 \%$ (PSP), and $32.5 \%$ (DN), respectively. To test whether Nestin could
A

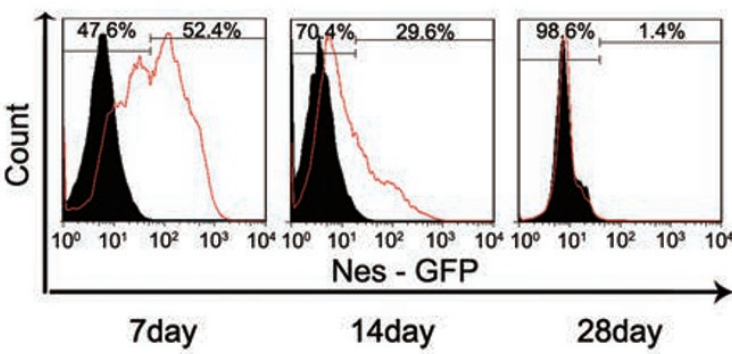

B
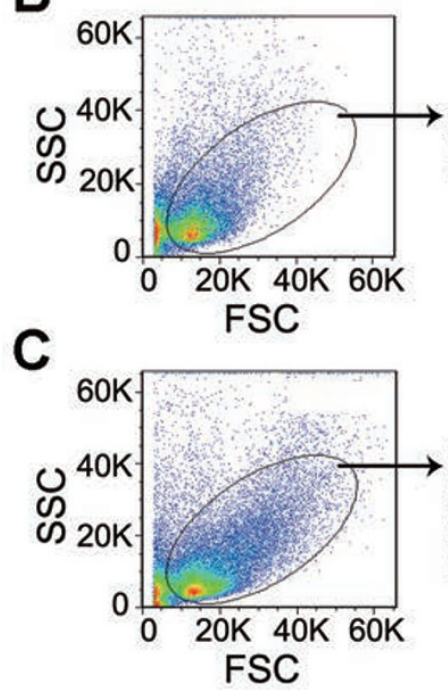
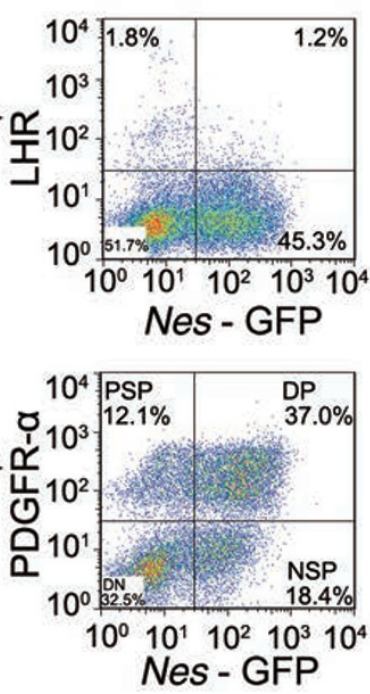
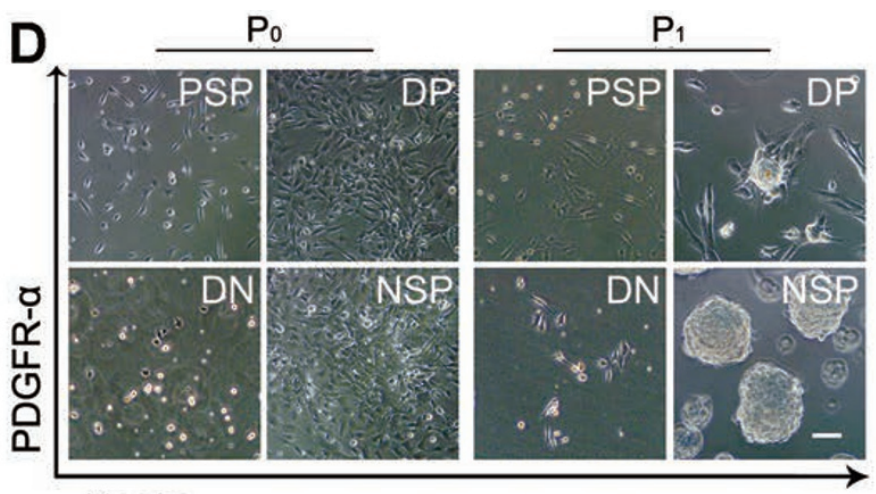

Nestin
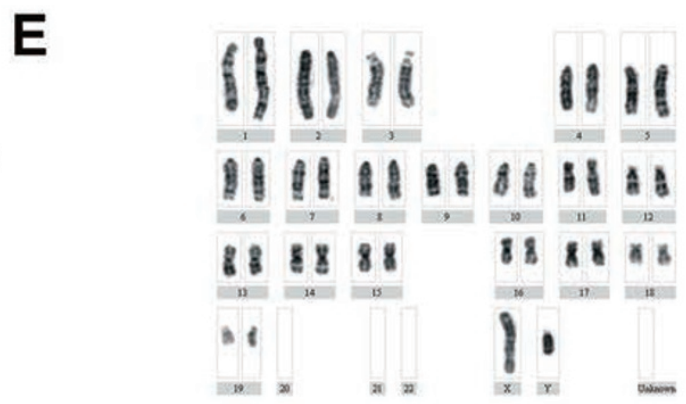

Figure 2 Isolation and phenotypic analysis of $\mathrm{Nes}_{-} \mathrm{GFP}^{+}$cells derived from the testis. (A) Flow cytometry was used to isolate Nes-GFP ${ }^{+}$cells from the testes of Nes-GFP transgenic mice on postnatal days 7, 14, and 28. The representative histogram overlays showed the isotype controls (black region) and the stained samples (red lines). $n=5$. (B, C) Representative flow cytometric profiles of Nes-GFP ${ }^{+}$cells stained with LHR (B) and PDGFR- $\alpha$ (C); these cells were derived from the testes of postnatal day 7 Nes-GFP transgenic mice. FSC, Forward-scattered light; SSC, Side-scattered light; PSP, PDGFR- $\alpha$ single positive; DP, Nes-GFP and PDGFR- $\alpha$ double positive; DN, double negative; NSP, Nes-GFP ${ }^{+}$single positive. (D) Phase-contrast micrographs of primary $\left(\mathrm{P}_{0}, 4\right.$ days after plating) and $\mathrm{P}_{1}$ (7 days after plating) cells in each population derived from the PSP, DP, DN, and NSP cells. Scale bar, $50 \mu \mathrm{m}$. (E) Cytogenetic analysis showed that the Nes-GFP cells at $\mathrm{P}_{25}$ have a diploid karyotype. 
be used as a marker of SLCs, we isolated cells in each subpopulation from freshly isolated testicular cells and cultured them in SLC expansion medium. One day after culturing in ultra-low adherence dishes with expansion medium, many cells in each group adhered to the plastic dish and presented a spindle-shaped morphology, except for the DN population. After being transferred into new flasks 7-10 days after the initial culturing, DP or NSP cells were able to form small spheres. Subsequently, most of the DP and NSP cells exhibited floating spheres and proliferated, resulting in larger spheres (Figure 2D). Notably, spheres derived from these two populations could be propagated approximately 50 times before their proliferative capacity decreased, probably as a consequence of cellular senescence. In contrast, PSP and DN cells did not proliferate for more than two passages and could not form floating spheres. Interestingly, the NSP cells also expressed PDGFR- $\alpha$ after three passages (data not shown). This result suggests that Nestin might be an earlier marker for SLCs compared with PDGFR- $\alpha$. The Nes-GFP ${ }^{+}$cells (including DP and NSP cells) were used in later experiments.

To examine the karyotype of these cells, we cytogenetically analyzed the Nes-GFP ${ }^{+}$cells at passages 25 and $50\left(\mathrm{P}_{25}\right.$ and $\left.\mathrm{P}_{50}\right)$. At $\mathrm{P}_{25}$, all cells showed a diploid chromosomal complement (Figure 2E). However, at $\mathrm{P}_{50}, 14 \%$ of the cells had hypodiploid or hyperdiploid chromosome numbers, ranging from 38-60 (data not shown). For subsequent experiments, we used $\mathrm{Nes}-\mathrm{GFP}^{+}$cells between passages 5-30.

Nes-GFP ${ }^{+}$cells exhibit self-renewal capacity and a high clonogenic potential

We characterized the cells of the Nes-GFP ${ }^{+}$spheres at $\mathrm{P}_{6}$ by immunofluorescence staining and examined the expression levels of PDGFR- $\alpha$ and LIFR, and embryonic stem cell markers, such as NANOG, OCT4, SOX2, SSEA-1 and SSEA-4. Interestingly, the Nes-GFP ${ }^{+}$sphere cells highly expressed Nestin and also expressed PDGFR- $\alpha$, LIFR, SSEA-1, and SSEA-4 (Figure 3A); however, they did not express OCT4 or NANOG and exhibited only negligible expression of SOX2 (data not shown). In addition, these isolated cells were negative for the LC markers LHR and 3 $\beta$-HSD, indicating that the culture conditions maintained the SLCs in their undifferentiated state. A majority of these cells were also positive for GATA4, which had been implicated as a key transcription factor in LC development $[18,19]$. Moreover, the Nes-GFP ${ }^{+}$cells were positive for the proliferation marker PH3 (Figure 3A). These results strongly suggest that Nes-GFP ${ }^{+}$cells have the characteristics of SLCs in the testis.
To demonstrate the self-renewal capacity of the Nes$\mathrm{GFP}^{+}$cells, we carried out a single-cell sphere formation assay in which single-cell suspensions derived from $\mathrm{P}_{7}$ Nes-GFP ${ }^{+}$cells were seeded by serial dilution into 96well plates (Figure 3B). Primary spheres appeared 4 days after plating, and the diameters of the spheres were larger than $50 \mu \mathrm{m}$ after 10 days of culture (Figure $3 \mathrm{C}$ ). The primary clonogenic efficiency of the $\mathrm{Nes}-\mathrm{GFP}^{+}$cells was $18.1 \% \pm 2.9 \%$. The seeding of single-cell suspensions from primary clones of $\mathrm{Nes}-\mathrm{GFP}^{+}$cells generated secondary clones that exhibited similar clonogenic efficiency (Figure 3D). Moreover, the proliferative potential of Nes$\mathrm{GFP}^{+}$cells was detected using the CCK8 assay, and their proliferation rates were similar at different passages $\left(\mathrm{P}_{7}\right.$, $\mathrm{P}_{15}$ and $\mathrm{P}_{30}$; Figure $\left.3 \mathrm{E}\right)$. These experiments revealed that Nes-GFP ${ }^{+}$cells were highly clonogenic and exhibited the capacity for self-renewal in vitro.

\section{Multilineage differentiation capacity of Nes-GFP cells in vitro}

Nestin has been identified as a marker for neural and mesenchymal stem cells, and these Nestin-expressing cells are capable of differentiating into neural [20] or mesenchymal [12] cell lineages, suggesting that Nestin represents a characteristic marker of multilineage progenitor cells. To characterize the multipotency of the $\mathrm{Nes}$-GFP ${ }^{+}$cells in the testis, we cultured these cells under defined differentiation conditions (Figure 4A). After induction, the neural lineage differentiation of $\mathrm{Nes}-\mathrm{GFP}^{+}$ cells was confirmed by staining for Tuj-1 (Neuronal class III $\beta$-tubulin) and GFAP (glial fibrillary acidic protein; Figure 4B and 4C). In addition, the induction of Tuj-1, neurofilament 200 (NF200) and GFAP gene expression was observed by RT-PCR analysis (Figure 4G). Osteogenic differentiation was confirmed by the deposition of alizarin red-positive mineralized matrix after 4 weeks of induction (Figure 4D). This increased calcium deposition was associated with increased mRNA levels of the osteogenic markers runt related transcription factor 2 (Runx2), secreted protein acidic and rich in cysteine (SPARC), and alkaline phosphatase (ALP; Figure 4G). Oil red O staining, which detects the presence of lipid-rich vacuoles, was used to evaluate the adipogenic induction (Figure $4 \mathrm{E})$. The differentiated cells exhibited intracellular lipid vacuoles that were absent in the undifferentiated cells. We further found that the expression levels of adipogenic markers including peroxisome proliferator activated receptor- $\gamma($ PPAR- $\gamma)$ and fatty acid-binding protein 4 (Fabp4) were significantly upregulated after differentiation (Figure 4G). Four weeks after chondrogenic induction, the pellet was intensely stained with toluidine blue (Figure 4F). Furthermore, in accordance with the 
A
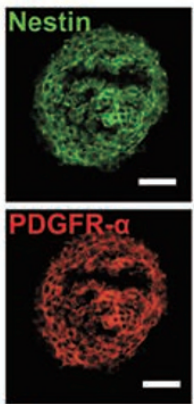

Merge

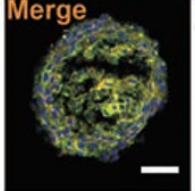

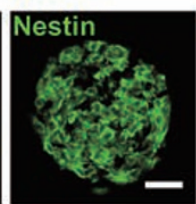
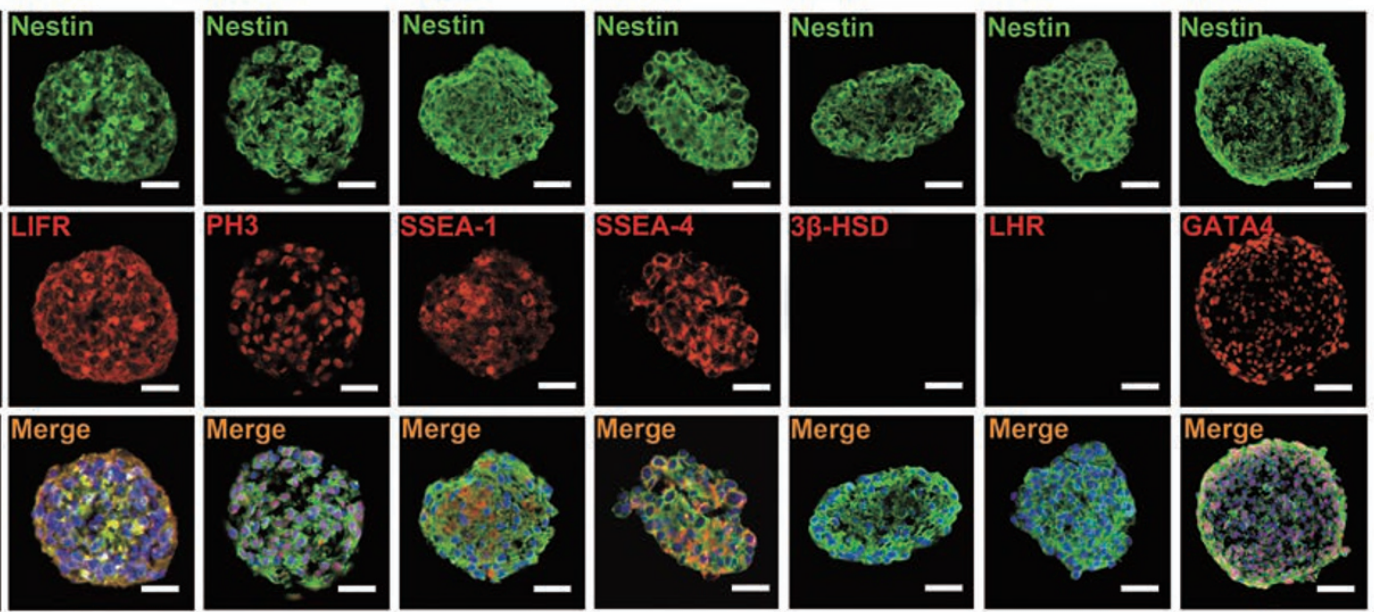

B
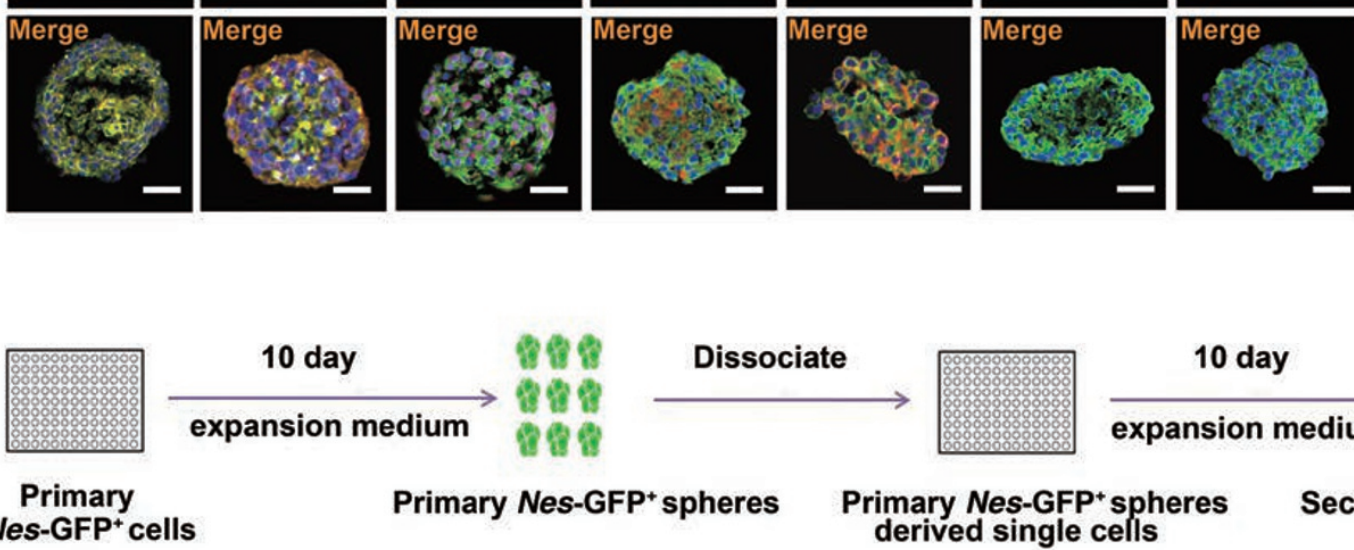

10 day

Primary Nes-GFP+spheres derived single cells

\section{Secondary Nes-GFP+ spheres}

C
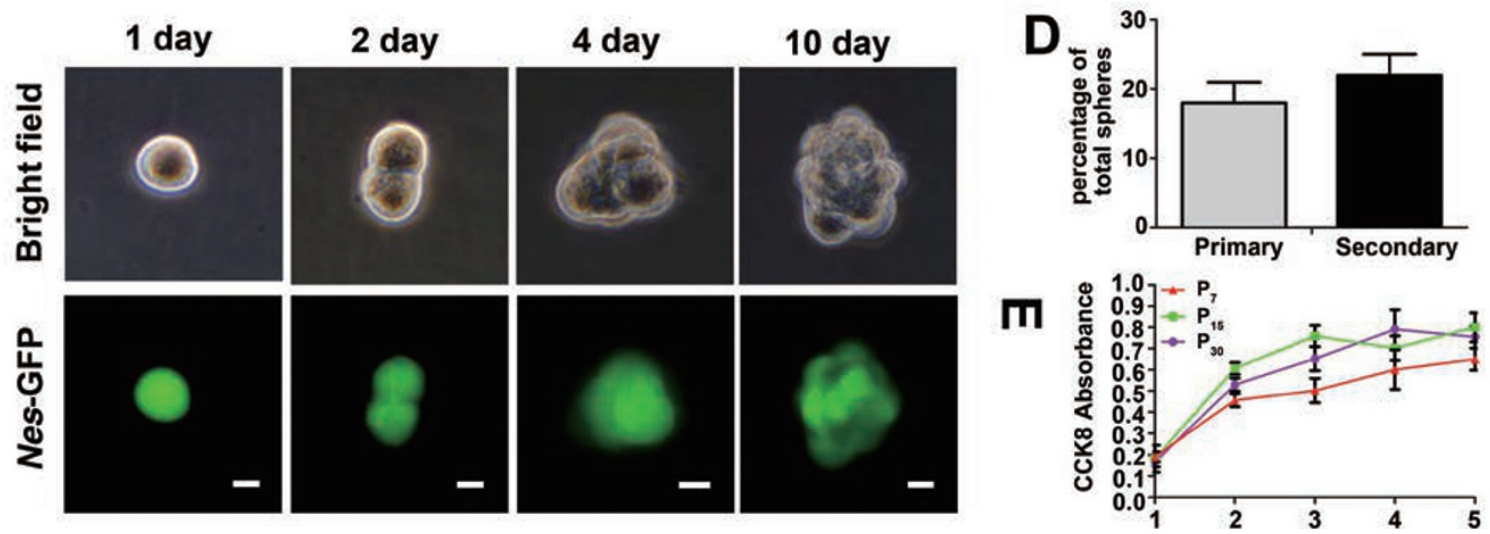

Figure $3 \mathrm{Nes} \mathrm{GFP}^{+}$cells showed self-renewal property. (A) Cultured Nes-GFP ${ }^{+}$spheres expressed PDGFR- $\alpha$, LIFR, PH3, SSEA-1, SSEA-4, and GATA-4, but did not express 3 $\beta-H S D$ or LHR. Nuclei were counterstained with DAPI (blue). Scale bar, $25 \mu \mathrm{m}$. (B) Schematic of the experimental procedure used to generate the Nes-GFP ${ }^{+}$clonal sphere cultures. (C) Representative images showed clonal sphere growth from single cells observed using a bright field (top panel) or fluorescence (bottom panel) microscope. Scale bar, $10 \mu \mathrm{m}$ (days 1 and 2) and $25 \mathrm{~m}$ (days 4 and 10). (D) A comparison of the sphere formation in wells containing a single cell showed that the frequency of sphere formation was equivalent in the primary and secondary spheres $(n=4)$. Data are expressed as the mean \pm SEM. (E) The proliferation rates of the Nes-GFP ${ }^{+}$cells at different passages $\left(P_{7}, P_{15}\right.$ and $\left.P_{30}\right)$ were assessed using the CCK8 assay and were shown to be similar $(n=6)$. Data are expressed as the mean \pm SEM.

histochemistry results, the mRNA expression levels of the chondrogenic genes Col2A and Aggrecan were also highly upregulated (Figure $4 \mathrm{G}$ ). It needs to be noted that the expression of these lineage-specific markers was not detected prior to differentiation (Figure $4 \mathrm{G}$ ).
To further assess the plasticity of the clonal (single cell-derived) Nes-GFP ${ }^{+}$cells, we performed the multilineage differentiation assay with colonies derived from a single Nes-GFP ${ }^{+}$cell (Figure 5A). The clonal Nes-GFP ${ }^{+}$ single cells were plated into 96 -well plates by serial dilu- 


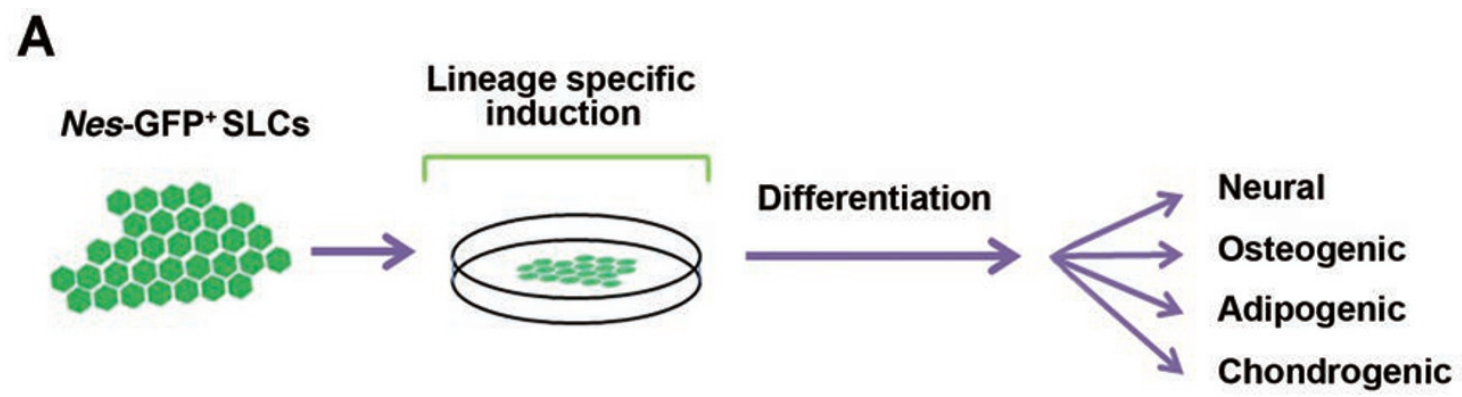

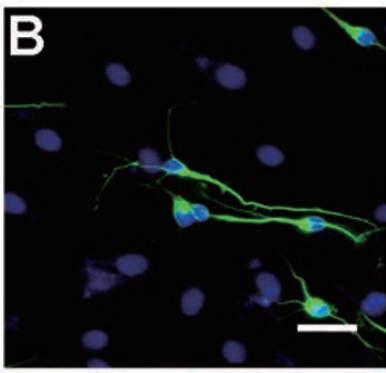
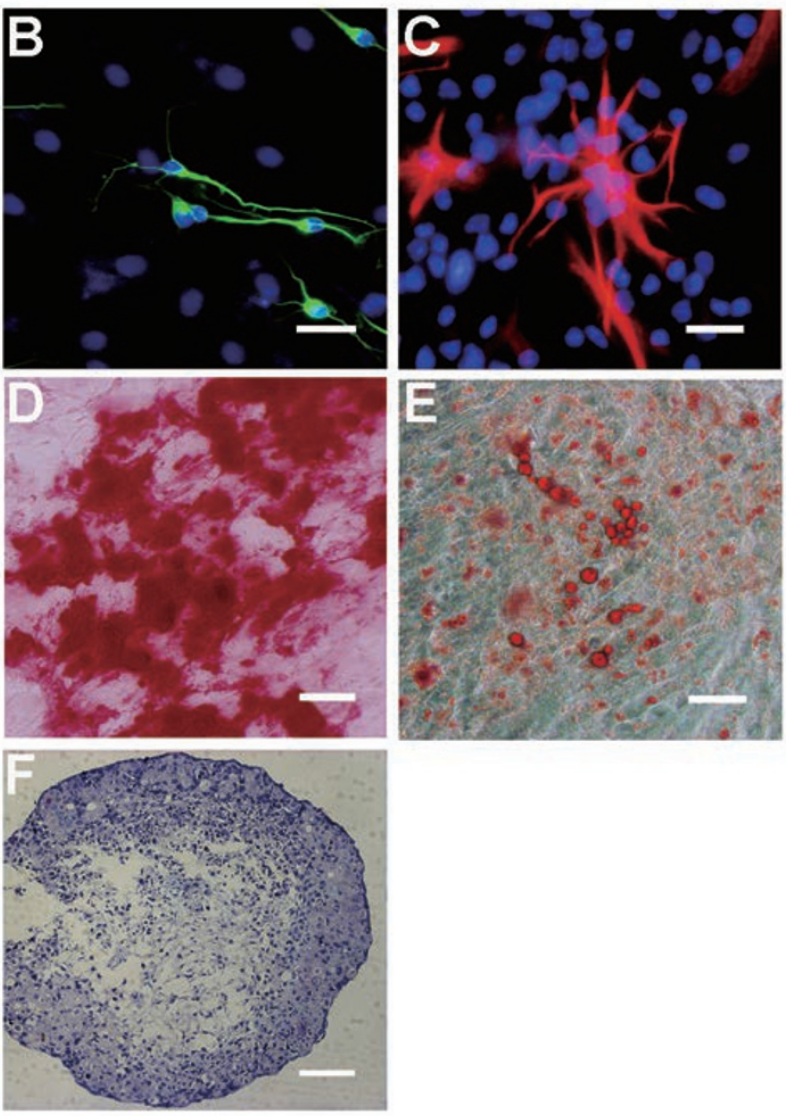
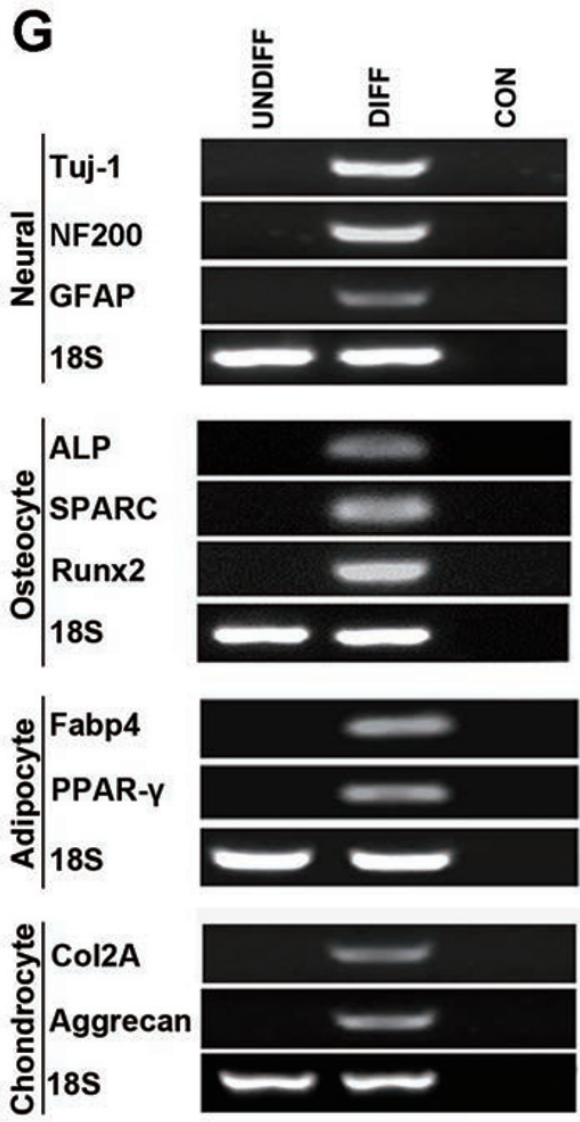

Figure 4 Nes-GFP ${ }^{+}$cells differentiated into multiple lineages. (A) Schematic of the experimental procedure used for inducing differentiation. (B-F) Representative micrographs of histological staining showing neurons (TuJ-1) (B), astrocytes (GFAP) (C), osteocytes (alizarin red) (D), adipocytes (oil red) (E), and chondrocytes (toluidine blue) (F). Scale bar, B-C, $25 \mu \mathrm{m}$; D-F, 100 $\mu \mathrm{m}$. (G) RT-PCR analysis of differentiated Nes-GFP ${ }^{+}$cells cultured for 4 weeks (DIFF) showing increased expression of neural (Tuj-1, NF200 and GFAP), osteogenic (ALP, SPARC and Runx2), adipogenic (Fabp4 and PPAR- $\gamma$ ), and chondrogenic (Col2A and Aggrecan) markers relative to undifferentiated SLCs (UNDIFF).

tion (one cell/well) and were expanded under non-adherent conditions. After treating the clonal $\mathrm{Nes}-\mathrm{GFP}^{+}$cells with differentiation media, these cells exhibited robust plasticity. Under the mesenchymal lineage differentiation conditions, these cells showed tri-lineage differentiation potential and exhibited osteogenic (Figure 5B and 5C), adipogenic (Figure 5B and 5D), and chondrogenic (Figure 5E) lineage phenotypes during the 4-week differentia- tion period. Interestingly, such clones also underwent neurogenic differentiation, as shown by the staining for the neuronal and astroglial proteins Tuj-1 and GFAP, respectively (Figure 5F and 5G). Moreover, these clonogenic experiments further suggest that these cells possess self-renewal potential, which was consistent with previous reports in which extensive proliferation of a single cell with the retention of multipotency over a long period 
A
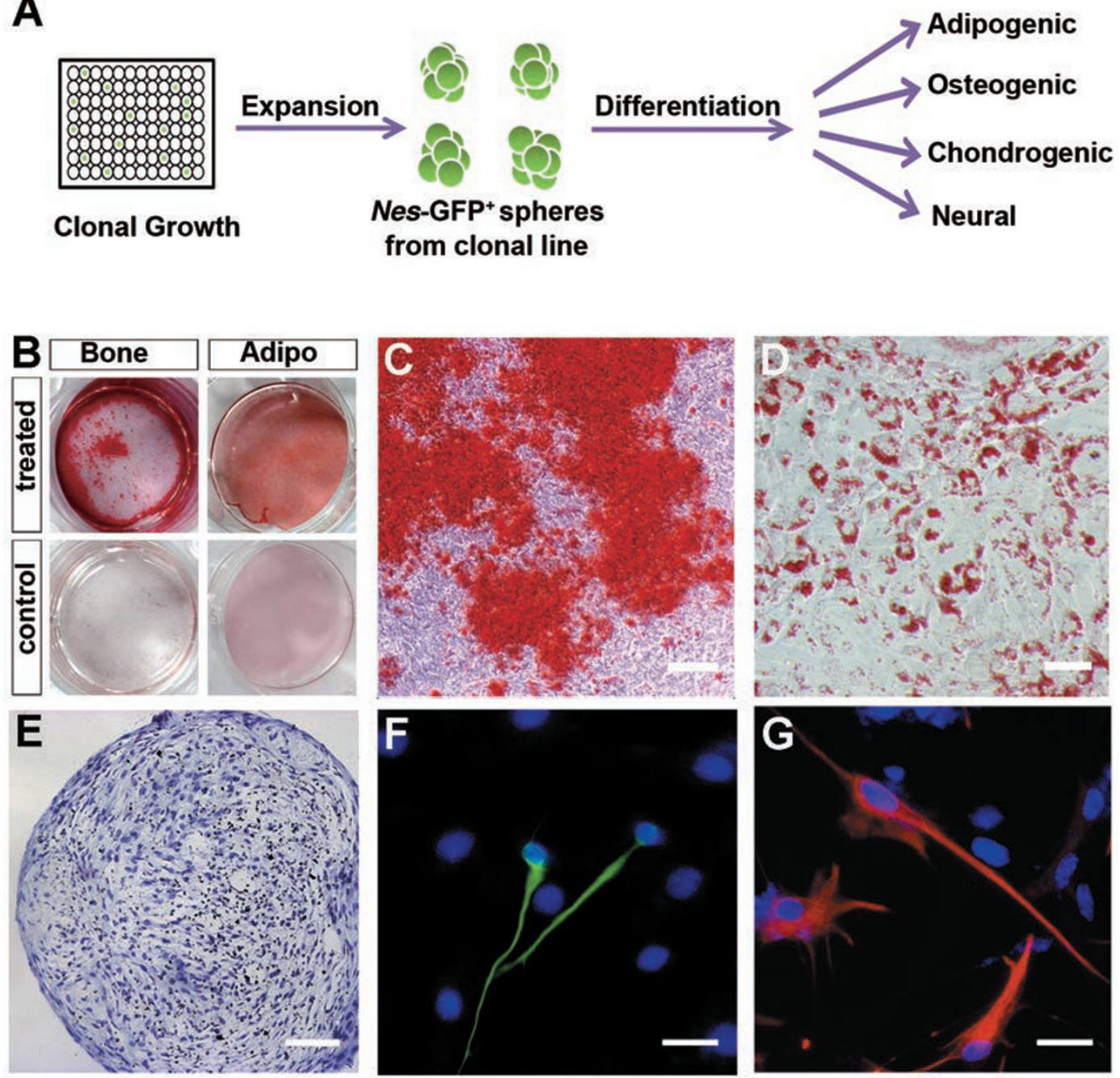

Figure 5 Clonal-derived Nes-GFP ${ }^{+}$cells exhibited multilineage differentiation capacity. (A) Schematic of the protocol for clonal Nes-GFP ${ }^{+}$cell differentiation. (B) Images of the Nes-GFP ${ }^{+}$clones under undifferentiated conditions (lower panels) and under osteogenic or adipogenic conditions (upper panels; left, alizarin red; right, oil red). (C-G) Single clones differentiated into osteocytes (alizarin red) (C), adipocytes (oil red) (D), chondrocytes (toluidine blue) (E), neurons (Tuj-1) (F), and astrocytes (GFAP) (G). Scale bar, C-E, $100 \mu \mathrm{m} ;$ F-G, $25 \mu \mathrm{m}$.

of time has been used to demonstrate stem cells in the skin [21].

\section{Nes-GFP ${ }^{+}$cells can differentiate into LCs in vitro}

To investigate the potential of the $N e s-\mathrm{GFP}^{+}$cells to differentiate into LCs in vitro, we placed the cells in the differentiation-inducing medium (DIM) previously reported by Ge et al. [9]. After 7 days of culture in DIM, numerous cells exhibited signs of LC differentiation, including the expression of proteins and enzymes involved in androgen biosynthesis. Immunohistochemical analysis showed that most of the differentiated Nes-GFP ${ }^{+}$cells expressed LC steroidogenic enzymes, including $3 \beta-\mathrm{HSD}$,
P450scc and P450c17, as well as LHR and steroidogenic acute regulatory protein (StAR). In addition, these cells were positively stained for the nuclear transcription factors, steroidogenic factor-1 (SF-1) [22] and GATA4 [18], which were known to be involved in LC development (Figure 6A). Moreover, RT-PCR demonstrated the upregulated expression of the LC lineage genes $3 \beta-\mathrm{HSD}$, StAR and LHR after 7 days of induction (Figure 6B). Next, to determine whether the differentiated $N e s-\mathrm{GFP}^{+}$cells could synthesize testosterone, we collected the supernatants at different time points and found that testosterone synthesis gradually increased, peaking 7 days after differentiation (Figure 6C). We also tested the steroidogenic 

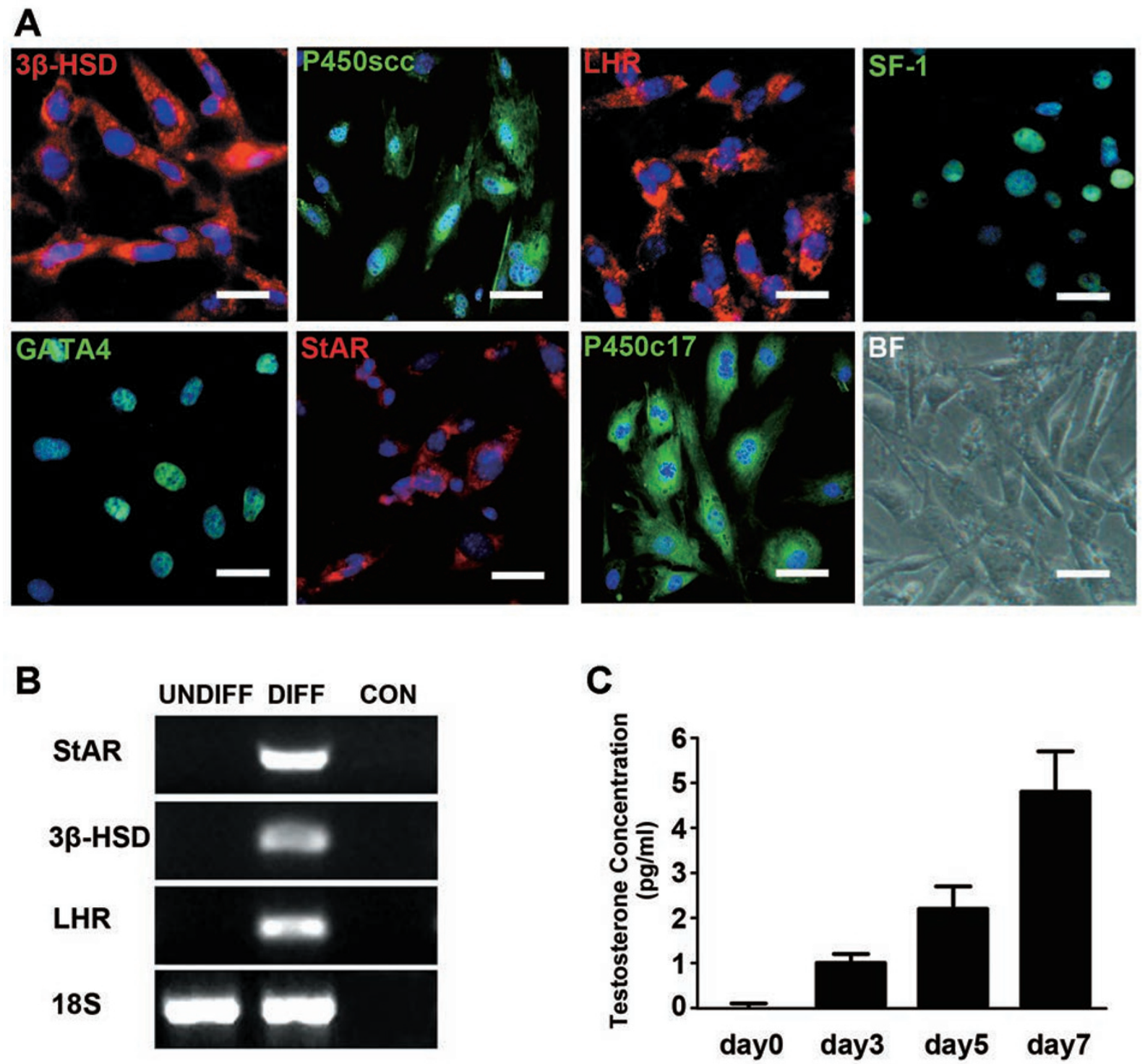

Figure 6 Nes-GFP ${ }^{+}$cells differentiated into LCs in vitro. (A) Seven days after differentiation, immunofluorescence staining showed that the Nes-GFP ${ }^{+}$cells clearly expressed LC lineage-specific markers. The representative images showed the expression of 33-HSD, P450scc, LHR, SF-1, GATA4, StAR and P450c17 in these cells. BF, bright field. Scale bar, $25 \mu \mathrm{m}$. (B) RT-PCR analysis confirmed that the expression of the Leydig lineage-specific markers StAR, 3 $\beta$-HSD, and LHR in differentiated cells (DIFF) was significantly increased while the undifferentiated controls (UNDIFF) exhibited undetectable levels of these markers. (C) Testosterone production progressively increased during culture in DIM. Data are expressed as the mean \pm $\operatorname{SEM}(n=6)$.

potential of the clonal Nes-GFP ${ }^{+}$cell lines derived from the Nes-GFP ${ }^{+}$single cells. Indeed, these cells successfully differentiated into LCs as demonstrated by the expression of LC lineage-specific markers as described above and the production of testosterone after differentiation (Supplementary information, Figure S1A and S1B). Taken together, these results suggest that $\mathrm{Nes}-\mathrm{GFP}^{+}$cells can differentiate into mature LCs capable of testosterone synthesis.

Transplanted Nes-GFP $\mathrm{Fells}^{+}$can colonize the interstitium of LC-disrupted or aging host animals and partially recover testosterone production

The ability to regenerate damaged tissues in vivo is an important property of stem cells. We therefore investigated whether the Nes-GFP ${ }^{+}$cells could differentiate 

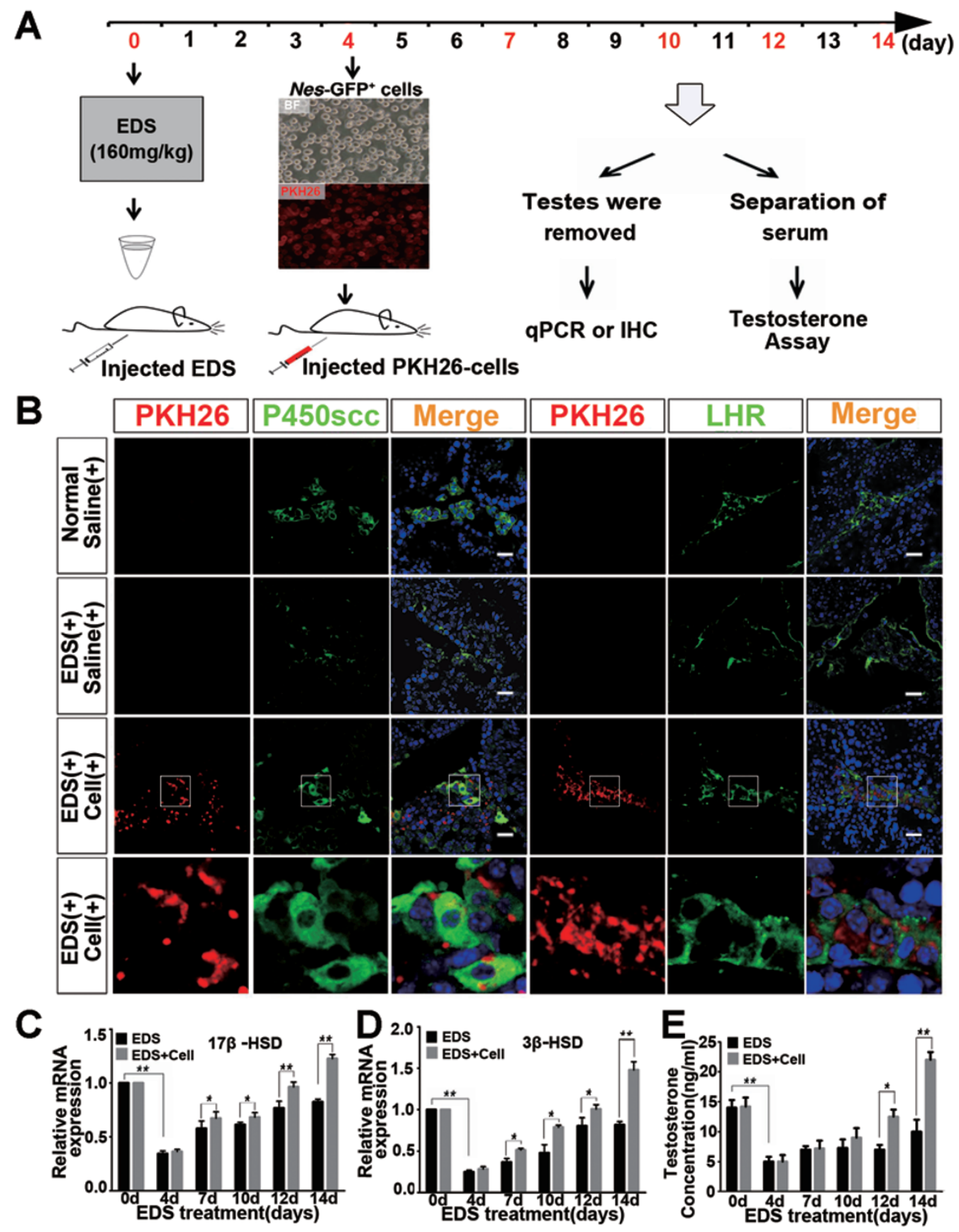

Figure 7 Transplanted Nes-GFP ${ }^{+}$cells differentiated into LCs that are capable of producing testosterone in the testes of EDS-treated 3-month-old mice. (A) Schematic of the experimental procedure used for cell transplantation. (B) Immunofluorescence staining showed the accumulation of cells positive for PKH26 (red) and P450scc (green) or LHR (green) in the interstitial area of the testis of EDS-treated mice 10 days after implantation with Nes-GFP ${ }^{+}$cells. In the control mice, the number of P450scc- and LHR-positive cells decreased after EDS injection. The bottom panels showed higher-magnification images of the dotted boxes in the lower-magnification images of the Nes-GFP ${ }^{+}$cell-transplanted mice. Scale bar, $50 \mu \mathrm{m}$. Normal/Saline (+), 3-month-old mice received saline injection; EDS(+)/Saline (+), EDS-treated mice receiving saline 4 days later; EDS(+)/Cells (+), EDS-treated mice receiving Nes-GFP ${ }^{+}$cells 4 days later. (C-D) qRT-PCR analysis showed the expression of $17 \beta-H S D(C)$ and $3 \beta-H S D(D)$ in the testes of or EDS- or EDS + Nes-GFP ${ }^{+}$cells-treated groups at the indicated experimental time points. Expression levels of each gene were compared to normal mice (before treatment; defined as 1). Data are shown as the mean \pm SEM. $n=6$. (E) The serum testosterone concentration was measured at the indicated time points in each animal. The level of testosterone was significantly increased in the Nes-GFP ${ }^{+}$cell-treated group compared to the control mice (treated with EDS alone) after cell transplantation $\left({ }^{\star} P<0.05,{ }^{* *} P<0.01\right)$. 
into the LCs and increase the testosterone levels in an LC-chemically disrupted model. Adult mice were treated with EDS $(160 \mathrm{mg} / \mathrm{kg})$, and then $\mathrm{Nes}-\mathrm{GFP}^{+}$cells were injected into the parenchyma at the cranial pole of the testis 4 days later. We observed that Nestin expression was significantly downregulated and GFP signals were also decreased dramatically after differentiation, which was in accordance with previous studies [12, 23] (Supplementary information, Figure S2A and S2B). Therefore, the Nes$\mathrm{GFP}^{+}$cells were labeled with PKH26 (a red fluorescent lipophilic dye), and implanted into recipient animals. At $0,4,7,10,12$, and 14 days after EDS treatment, the serum and the testes were collected for analyses (Figure 7A). The transplanted PKH26-labeled Nes-GFP ${ }^{+}$cells were localized exclusively to the interstitium of the testis, and expressed the LC-specific markers P450scc and LHR 10 days after transplantation (Figure 7B). Moreover, after EDS treatment, the expression of $17 \beta-\mathrm{HSD}$ and $3 \beta-H S D$, and the concentration of testosterone were decreased significantly on day 4 and recovered gradually (Figure 7C-7E). However, EDS treatment had no obvious effect on LC morphology (Figure 7B). Furthermore, the transplantation of $\mathrm{Nes}$-GFP $\mathrm{GP}^{+}$cells significantly increased the mRNA expression levels of LC-specific markers, $3 \beta$-HSD and $17 \beta$-HSD, and the production of testosterone in EDS-treated mice at different time points (Figure 7C-7E). On day 14 (day 10 after cell transplantation), the testosterone concentration was even higher than that of saline-injected normal mice (Figure 7E). We then isolated PKH26-labeled cells by FACS from the testis 10 days after transplantation and performed RTPCR analysis, which revealed that these cells expressed LC lineage-specific markers including LHR, 17 $\beta$-HSD and 3 $\beta$-HSD (Supplementary information, Figure S3A). Although GFP gene was not expressed at the transcriptional level after differentiation, it should be still located in the genomic DNA as an exogenous gene. Indeed, we found that most PKH26-labeled cells from the mouse testis carried the GFP gene (Supplementary information, Figure S3B).

To further confirm the function of these cells, we established the EDS-treated rat model [9] and injected Nes-GFP ${ }^{+}$cells into the parenchyma of the rat testis. Consistent with previous observation [14], in EDS-treated control group, the P450scc-positive cells were almost not observed in the interstitium, and serum testosterone levels were dramatically decreased 14 days after EDS treatment, indicating that EDS specifically eliminated the testosterone-producing LCs in the adult rat model. Importantly, we found that the transplanted PKH26-labeled $\mathrm{Nes}-\mathrm{GFP}^{+}$cells were localized in the interstitium and expressed P450scc and LHR, and that the cell trans- plantation partially restored testosterone production (Supplementary information, Figures S4 and S5). These data suggest that the transplanted $\mathrm{Nes}-\mathrm{GFP}^{+}$cells have differentiated into functional LCs in vivo.

In male mice, aging generally goes along with a progressive decline in the biosynthesis of testosterone by LCs [24-26]. To test whether Nes-GFP ${ }^{+}$cells could restore testosterone production in a senescence model, we injected PKH26-labeled Nes-GFP ${ }^{+}$cells into the testes of 22-month-old mice. Without cell transplantation, the expression levels of P450scc and LHR in the LCs of these old mice were dramatically decreased (Figure 8A), and these mice also exhibited a lower serum testosterone level compared with young adults injected with saline (Figure 8B). Ten days after cell transplantation, the PKH26-labeled Nes-GFP ${ }^{+}$cells colonized the interstitium of the testis and strongly expressed P450scc and LHR (Figure $8 \mathrm{~A})$. Notably, the serum testosterone levels of mice with cell transplantation were increased significantly compared with the control mice with saline injection (Figure 8B). Taken together, these results demonstrate that the $\mathrm{Nes}-\mathrm{GFP}^{+}$cells possess key properties of SLCs as they are capable of colonizing the interstitium and differentiating into mature testosterone-producing LCs.

\section{Transplanted Nes-GFP cells offer potential benefits for spermatogenesis}

It has been reported that spermatogenic dysfunction is often associated with impaired LC function [27]. Furthermore, it has been established that testosterone is essential for spermatogenesis [28]. To further verify whether the transplanted Nes-GFP ${ }^{+}$cells played a role in the maintenance of spermatogenesis, we examined the proliferative capacity of spermatogonial cells in the seminiferous tubules. PH3-positive cells virtually disappeared in the EDS-treated mice (Figure 9A), whereas the number of PH3-positive proliferating cells was increased dramatically in the seminiferous tubules 10 days after transplantation with the $N e s-\mathrm{GFP}^{+}$cells $(0.33 \% \pm 0.11 \%$ versus $21.97 \% \pm 2.49 \%, P<0.01$, Figure 9B). More interestingly, in the testes of aging mice, $\mathrm{PH} 3$ expression was rarely detected in the seminiferous tubules $(2.40 \% \pm 0.58 \%)$, whereas the number of proliferating spermatogenic cells was obviously increased after Nes-GFP ${ }^{+}$cell transplantation $(2.40 \% \pm 0.58 \%$ versus $10.87 \% \pm 1.01 \%, P<0.01$, Figure 9B).

Previous studies have demonstrated that testosterone action is critical for the completion of meiosis and spermiogenesis in rodents $[29,30]$, we thereby detected the expression of meiotic markers, synaptonemal complex protein 1 and 3 (SYCP1 and SYCP3) [31, 32], by immunofluorescence staining (Figure 9C and 9E). We observed 


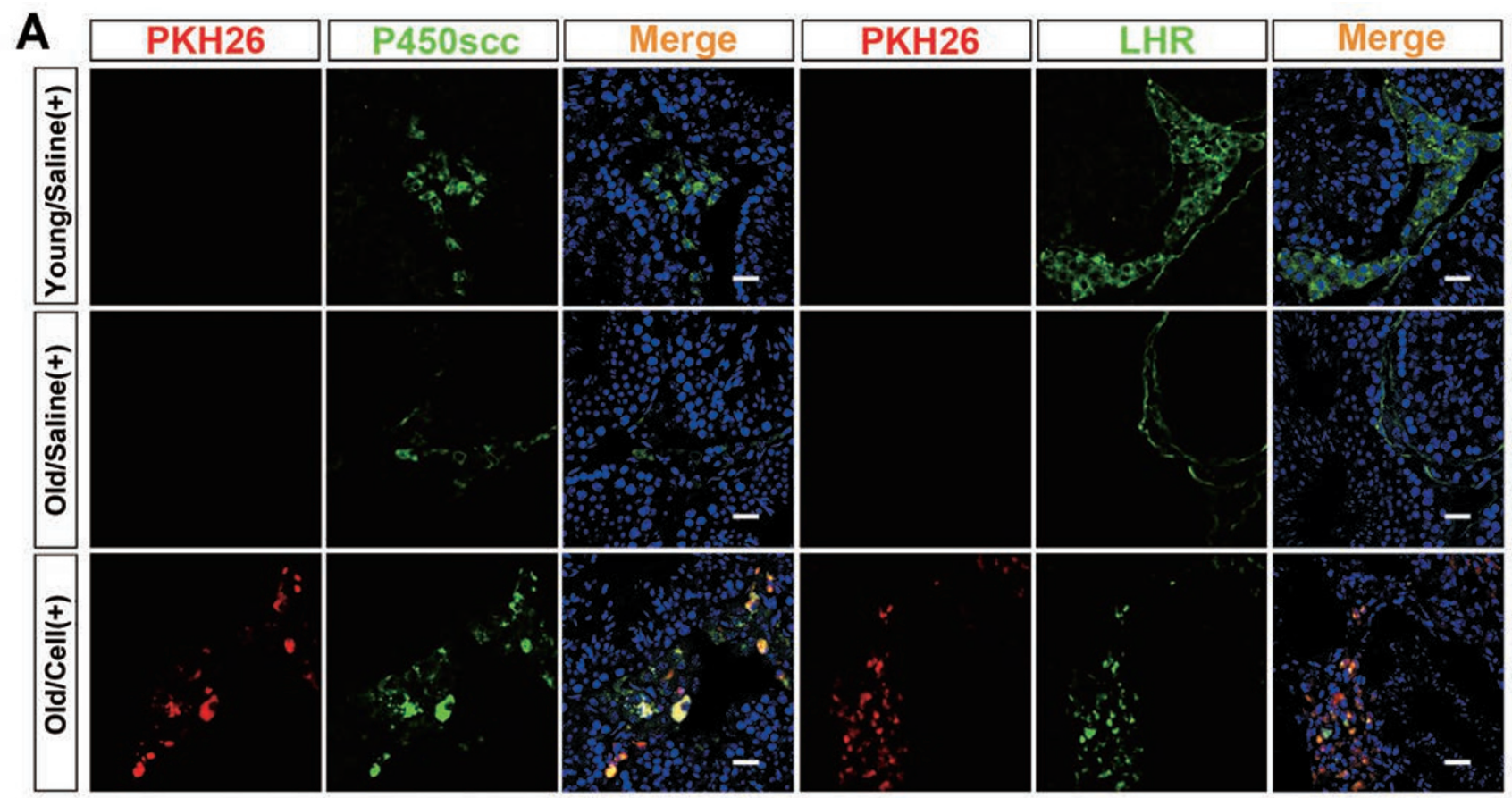

B

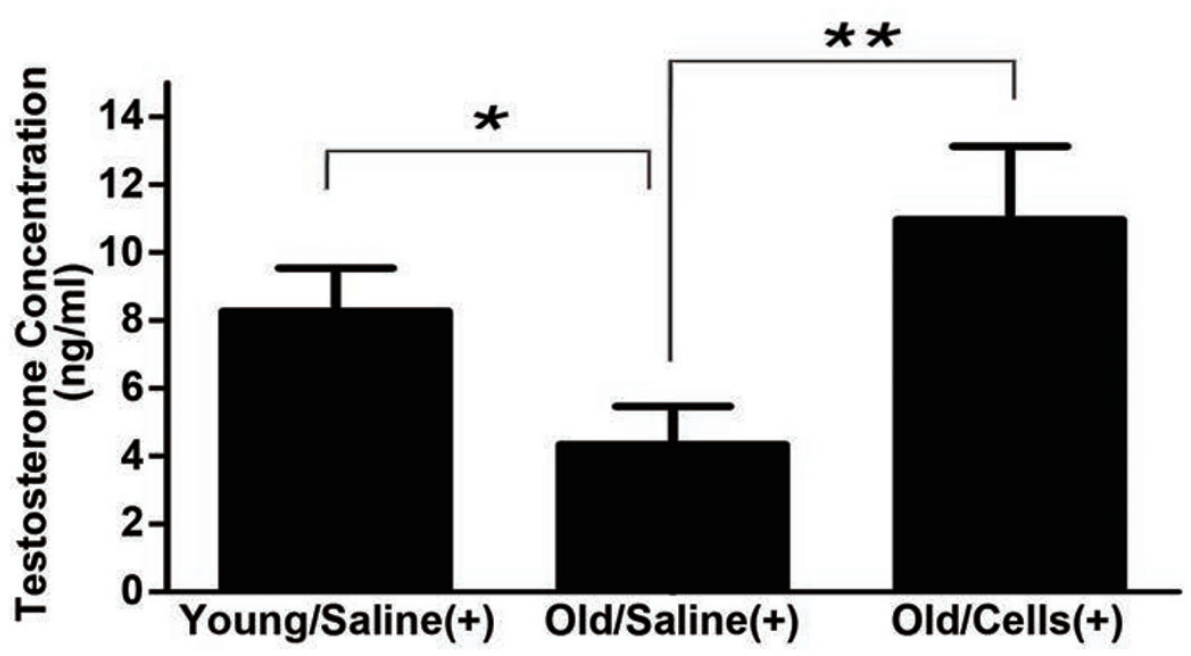

Figure $8 \mathrm{Nes}^{-G F P^{+}}$cells differentiated into LCs and facilitated testosterone production in the testes of 22-month-old mice after transplantation. (A) Immunofluorescence staining showed the accumulation of cells positive for PKH26 (red) and P450scc (green) or LHR (green) in the interstitial area of testes of old mice 10 days after Nes-GFP ${ }^{+}$cell implantation. The expression levels of P450scc and LHR in the 22-month-old mice were lower than those in the young mice (3-month-old). Scale bar, $50 \mu \mathrm{m}$. (B) In the 22-month-old mice, the serum testosterone concentrations were significantly increased in the Nes-GFP cell-treated mice compared to the saline-treated old mice $\left({ }^{*} P<0.05,{ }^{* *} P<0.01\right) . n=6$. Young/Saline $(+)$, 3-month-old mice receiving saline injection; Old/Saline (+), 22-month-old mice receiving saline injection; Old/Cells (+), 22-month-old mice receiving Nes-GFP+ cells.

that SYCP1 - (Figure 9C) or SYCP3-positive (Figure 9E) cells were significantly decreased in EDS-treated mice. However, after transplantation with $\mathrm{Nes}-\mathrm{GFP}^{+}$cells, the meiotic cells dramatically increased (SYCP1: 7.19\% $\pm 1.21 \%$ versus $60.32 \% \pm 5.10 \%, P<0.01$; SYCP3 :
$15.10 \% \pm 2.01 \%$ versus $69.10 \% \pm 6.08 \%, P<0.01)$. The similar phenomena were observed in the aging mice treated with Nes-GFP ${ }^{+}$cells (SYCP1: $18.35 \% \pm 1.91 \%$ versus $31.82 \% \pm 5.53 \%, P<0.01 ; \mathrm{SYCP} 3: 25.12 \% \pm$ $3.20 \%$ versus $67.80 \% \pm 3.09 \%, P<0.01$, Figure 9D and 
A

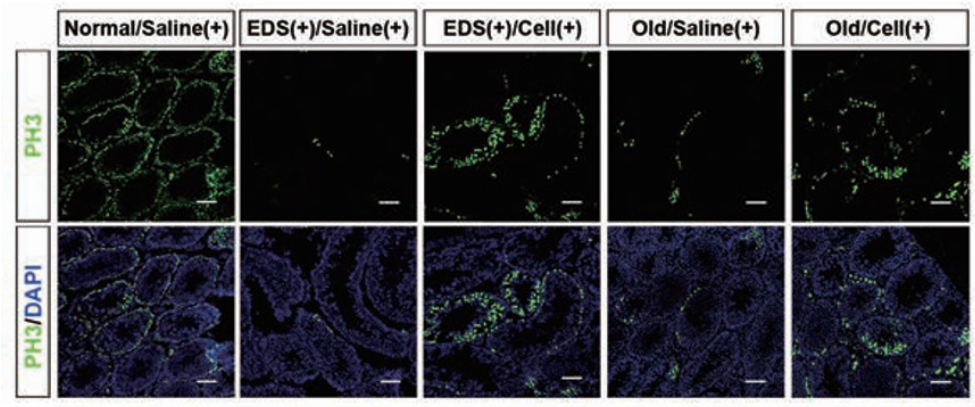

\section{C}
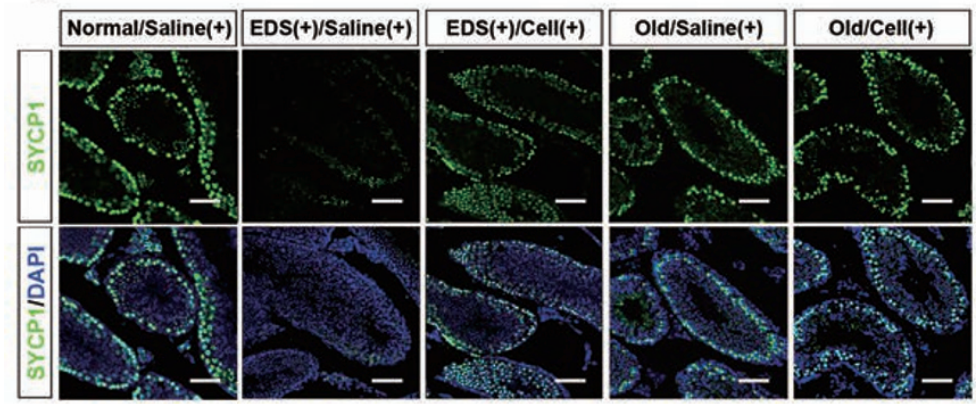

E

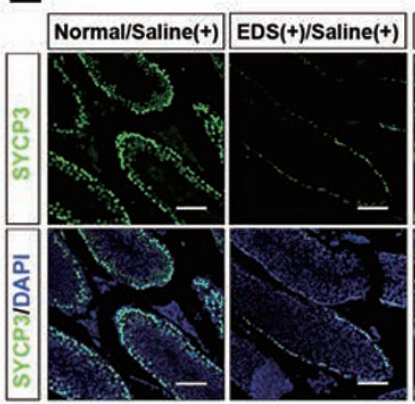

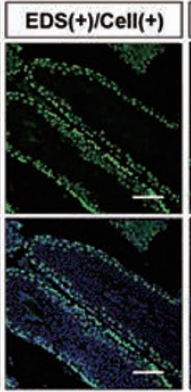

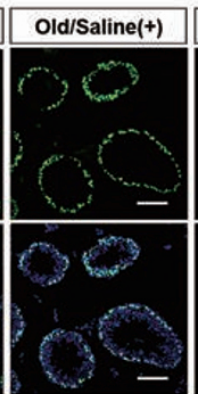

B

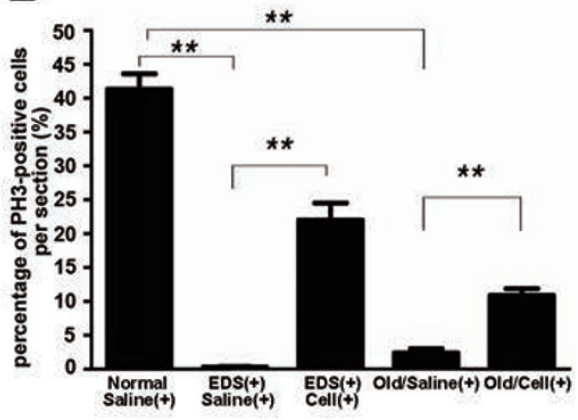

D

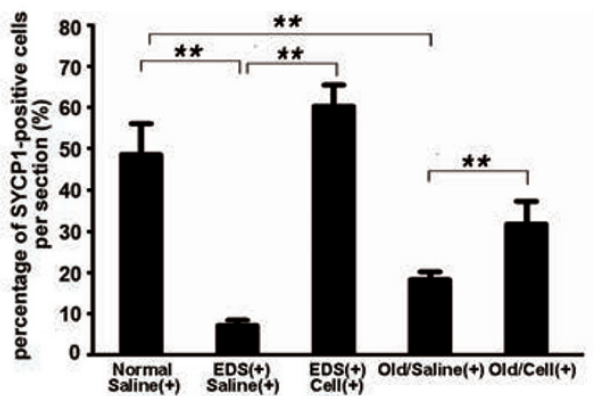

$\mathbf{F}$

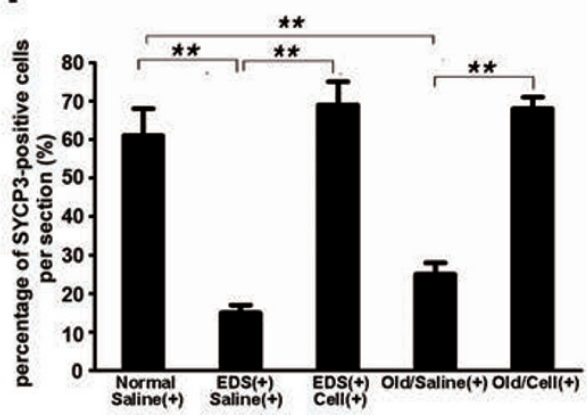

Figure 9 Nes-GFP ${ }^{+}$cells promoted spermatogenesis 10 days after transplantation. (A) Representative images showed PH3positive (green) cells in seminiferous tubules of testes in each experimental mouse. Ten days after transplantation, the Nes$\mathrm{GFP}^{+}$cell-treated animals showed a significantly higher number of proliferating cells in the seminiferous tubules compared to mice with saline injection. Scale bar, $50 \mu \mathrm{m}$. (B) Quantitative analysis showing the percentage of PH3-positive cells in seminiferous tubules of testes. Three sections per slide and three slides per mouse testis were counted $\left({ }^{\star \star} P<0.01\right)$. (C, E) The meiotic spermatocytes were observed by immunofluorescence staining with anti-SYCP1 (green) (C) and anti-SYCP3 (green) (E) antibodies. Nucleus was detected by DAPI staining (blue). The SYCP1- or SYCP3-positive cells were located at the pachytene stage in each testis. Scale bar, $50 \mu \mathrm{m}$. (D, F) Quantitative analysis showing the percentage of SYCP1or SYCP3-positive cells in seminiferous tubules of testis. Three sections per slide and three slides per mouse testis were counted $\left({ }^{* *} P<0.01\right)$. Normal/Saline $(+)$, 3-month-old mice receiving saline injection; EDS $(+) /$ Saline $(+)$, EDS-treated mice receiving saline 4 days later; EDS $(+) / C e l l s(+)$, EDS-treated mice receiving Nes-GFP ${ }^{+}$cells 4 days later; Old/Saline $(+)$, 22-month-old mice receiving saline injection; Old/Cells (+), 22-month-old mice receiving Nes-GFP ${ }^{+}$cells. $n=6$.

9F). In addition, the increase of meiotic cells was also observed in EDS-treated rat model 10 days after Nes$\mathrm{GFP}^{+}$cell transplantation (Supplementary information, Figure S6A and S6B).

$H \& E$ histological analysis further revealed that the transplantation of Nes-GFP ${ }^{+}$cells increased the number of round spermatids in the EDS-treated animals and aging mice (Supplementary information, Figure S7). Taken together, these results demonstrate that the transplantation of $\mathrm{Nes}-\mathrm{GFP}^{+}$cells can partially restore the disrupted 
LC function, leading to the production of biologically active levels of testosterone, which may improve spermatogenesis.
CD51 is highly expressed on Nes-GFP $P^{+}$cells and CD51 cells exhibit the characteristics of SLCS

As Nestin is an intracellular protein, it is useful to
A

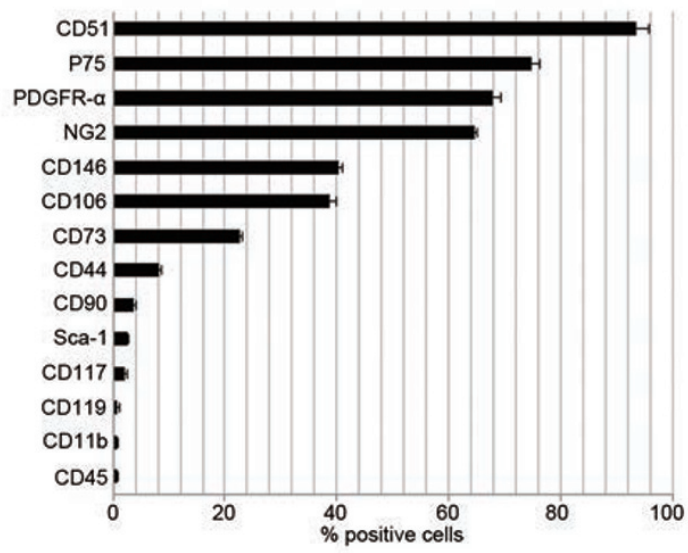

B
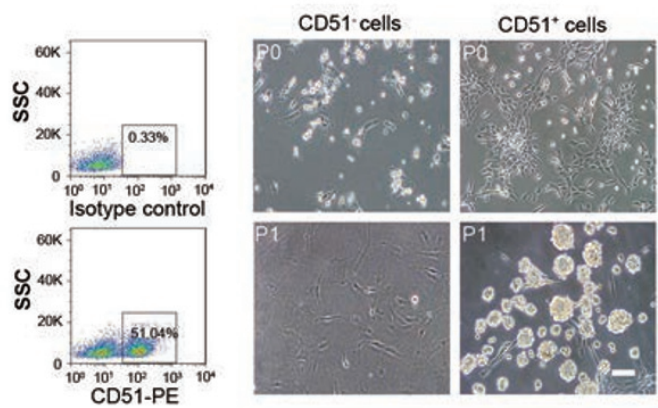

C
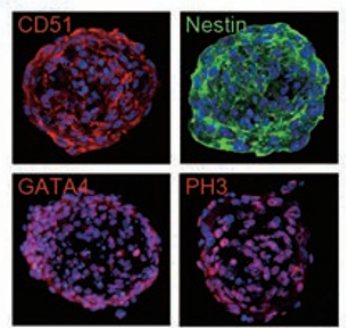
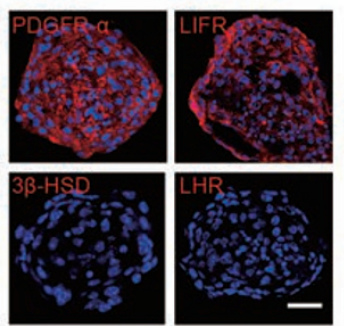

D

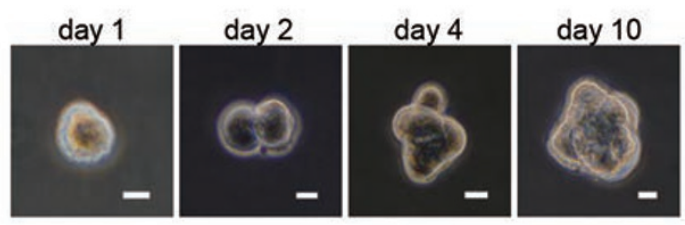

E
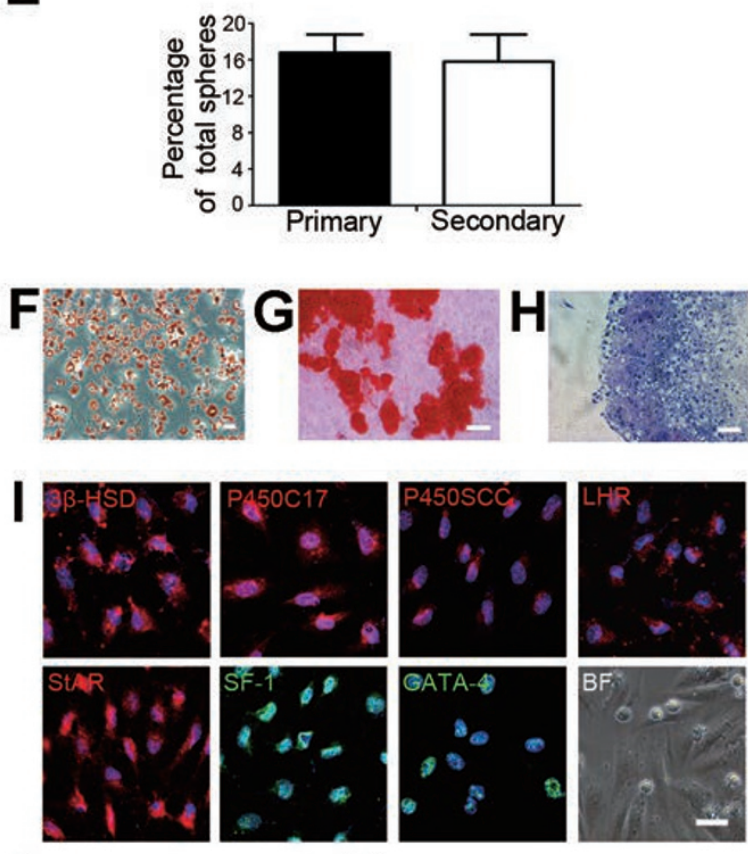

J

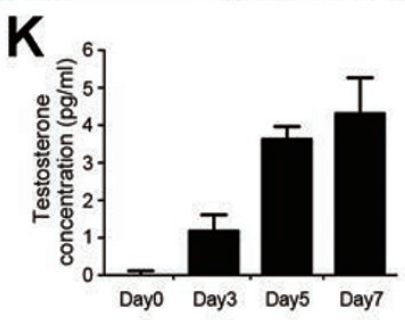

Figure $10 \mathrm{CD} 51^{+}$cells exhibited the characteristics of SLCs. Primary Nes-GFP ${ }^{+}$cells were isolated from postnatal 7-day-old Nes-GFP transgenic mice and the expression of the indicated cell surface markers was analyzed by flow cytometry. (B) Representative flow cytometric profiles of $\mathrm{CD} 51^{+}$cells in testis of postnatal 7-day-old C57BL/6 mice. Phase-contrast micrographs showed that primary $\left(\mathrm{P}_{0}, 4\right.$ days after plating $)$ and $\mathrm{P}_{1}\left(7\right.$ days after plating) cells in both $C D 51^{+}$and $C D 51^{-}$cells. Scale bar, 25

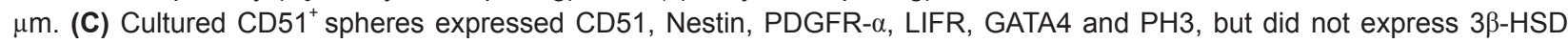
or LHR. Nuclei were counterstained with DAPI (blue). Scale bar, $25 \mu \mathrm{m}$. (D) Representative images showing clonal sphere growth derived from single cells, which were observed by bright field microscopy. Scale bar, $10 \mu \mathrm{m}$ (days 1 and 2 ) and $25 \mu \mathrm{m}$ (days 4 and 10). (E) Frequency of sphere formation from single cell-derived CD51 $1^{+}$cells was equivalent in the primary and secondary spheres $(n=4)$. Data are expressed as the mean \pm SEM. $(F-H)$ Representative images showed that clonally expanded CD51 $1^{+}$cells differentiated into adipocytes (oil red) (F), osteocytes (alizarin red) (G), and chondrocytes (toluidine blue)

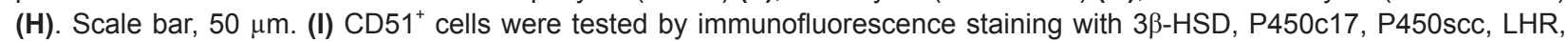
StAR, SF-1 and GATA4 7days after differentiation in DIM. BF, bright field. Scale bar, $25 \mu \mathrm{m}$. (J) Differentiated CD51 ${ }^{+}$cells

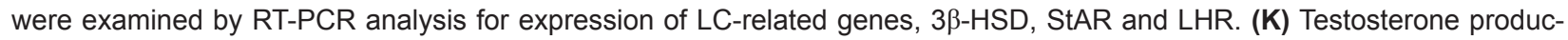
tion was measured in culture medium $0,3,5,7$ days after LC differentiation. Data are expressed as the mean \pm SEM. $n=6$. 
identify cell surface markers specific for $\mathrm{Nes}^{+}$SLCs. We screened the expression of the previously reported neural and mesenchymal stem cell surface markers in Nes$\mathrm{GFP}^{+}$cells derived from testes of postnatal 7-day-old mice, including CD51, p75 (CD271), PDGFR- $\alpha$, NG2, CD146, CD106, CD73, CD44, CD90, Sca-1, CD117, CD119, CD11b, and CD45 through flow cytometry. CD51 and p75 were highly expressed in Nes-GFP ${ }^{+}$cells $(93.53 \% \pm 3.10 \%$ and $74.73 \% \pm 1.89 \%$, respectively). Another putative SLC marker, PDGFR- $\alpha$ was expressed by $67.89 \% \pm 2.83 \%$ of $\mathrm{Nes}-\mathrm{GFP}^{+}$cells. Moreover, two known perivascular pericyte markers, NG2 [33] and CD146 [34], were expressed in $64.63 \% \pm 3.16 \%$ and $40.38 \% \pm 2.76 \%$ of $\mathrm{Nes}-\mathrm{GFP}^{+}$population, respectively. Furthermore, the expression of other conventional mesenchymal lineage markers (CD106, CD73, CD44, CD90, Sca-1, and CD117) was detected in a low percentage of $\mathrm{Nes}-\mathrm{GFP}^{+}$cells $(<40 \%)$. Moreover, Nes-GFP ${ }^{+}$cells lacked the expression of hematopoietic cell markers, CD11b and CD45 (Figure 10A and supplementary information, Figure S8).

Based on the highest level of CD51 expressed by Nes$\mathrm{GFP}^{+}$cells, we then tested whether CD51 could be a candidate marker for SLCs. CD51 ${ }^{+}$or CD51 ${ }^{-}$cells were sorted from testes of postnatal 7-day-old C57BL/6 mice by FACS and cultured in SLC expansion medium. One day after culturing in ultra-low adherence dishes, $\mathrm{CD} 51^{+}$cells adhered to the plastic, presented a spindle-shaped morphology and then were able to form non-adherent primary spheres 7 days after first passage, displaying a similar phenotype to Nes-GFP ${ }^{+}$cells (Figure 10B). In contrast, $\mathrm{CD} 1^{-}$cells did not proliferate for more than two passages. In addition, immunofluorescence staining further demonstrated that $\mathrm{CD} 51^{+}$cells also highly expressed Nestin, PDGFR- $\alpha$, LIFR, GATA4 and PH3, but did not express the LC markers, $3 \beta$-HSD and LHR (Figure 10C). To demonstrate the self-renewal capacity of the CD $51^{+}$ cells, we carried out a single-cell sphere formation assay in which single-cell suspensions derived from $\mathrm{P}_{5}$ were used. The primary and secondary clonogenic efficiency of the CD51 $1^{+}$cells were $16.89 \% \pm 2.09 \%$ and $15.78 \% \pm$ $3.22 \%$, respectively (Figure $10 \mathrm{D}$ and $10 \mathrm{E}$ ). These results suggest that similar to $\mathrm{Nes}-\mathrm{GFP}^{+}$cells, $\mathrm{CD} 51^{+}$cells were highly clonogenic and exhibited the capacity of self-renewal in vitro.

Furthermore, clonally expanded $\mathrm{CD} 51^{+}$cells were induced to differentiate under lineage-specific differentiation conditions, these cells showed robust differentiation potential, including adipogenic, osteogenic, and chondrogenic ability (Figure 10F-10H). Notably, in the LC differentiation medium, $\mathrm{CD} 51^{+}$cells efficiently differentiated into LCs as shown by the expression of $3 \beta-\mathrm{HSD}$,
P450c17, P450scc, LHR, StAR, SF-1, and GATA4 (Figure 10I). Moreover, RT-PCR analysis demonstrated the upregulated expression levels of the LC lineage genes $3 \beta$-HSD, StAR, and LHR (Figure 10J). In addition, testosterone production progressively increased and peaked on day 7 (Figure 10K). Taken together, these results suggest that CD51 can serve as a specific cell surface marker for SLCs.

\section{Discussion}

In this study, we sought to examine whether Nestin can serve as a novel marker for the identification and isolation of SLCs. We isolated Nes-GFP ${ }^{+}$cells from the testes of Nes-GFP transgenic mice, and found that these cells had characteristics consistent with SLC populations isolated by conventional methods $[9,10]$. Nes-GFP ${ }^{+}$cells not only proliferated continuously, but also showed highly clonogenic potential, which suggests that Nestin-positive SLCs were capable of self-renewal. Moreover, Nestin-positive SLCs not only were capable of differentiation into testosterone-producing LCs, but also showed the neural and mesenchymal lineage differentiation potential. In addition, when transplanted into the testes of LC-disrupted or aging animal models, the $N e s-\mathrm{GFP}^{+}$cells could colonize the interstitium, differentiate into LCs, partially recover testosterone production and improve spermatogenesis. Our findings provide new insight into the development of cell replacement therapies for the treatment of the patients with testosterone deficiency or decline.

The intermediate filament protein Nestin is a widely employed marker of multipotent neural stem cells (NSCs), and we have previously shown that Nestin is important for the survival and self-renewal of NSCs during embryonic development [35]. In adults, Nestin expression is frequently detected in areas of regeneration, such as neural progenitors, mesenchymal stem cells, hair follicle stem cells and myogenic cells, where Nestin-positive cells might function as a reservoir of stem/progenitor cells capable of proliferation and differentiation. Davidoff et al. [14] first proposed that Nestin might be a candidate marker for LC progenitor cells, but this study did not demonstrate that isolated cells are capable of self-renewal and differentiation in vitro. Previous studies have reported methods of isolating SLCs, including selection based on side population [8], Percoll gradient centrifugation, and cell surface marker expression [9]. However, these approaches have limitations: only a small number of cells can be isolated, and these methods can result in heterogeneous populations. To overcome these hurdles, we tried a different approach. We first demonstrated that the expression pattern of Nestin largely over- 
lapped with that of the putative SLC markers PDGFR- $\alpha$ and LIFR in the testis; therefore, the Nes-GFP ${ }^{+}$cells were selected on the basis of GFP expression using FACS. These cells could be expanded in culture for months, and displayed all of the characteristics of SLCs. Collectively, we demonstrated a simple and efficient approach to prospectively isolate SLCs.

Remarkably, under appropriate culture conditions, both non-clonal (non-single cell-derived) and clonal (single cell-derived) Nes-GFP ${ }^{+}$SLCs could differentiate into steroidogenic adult LCs capable of testosterone synthesis. Additionally, these cells could differentiate into neural and mesenchymal lineage cell types, such as neurons, astrocytes, osteoblasts, adipocytes, and chondrocytes. The clonogenicity and multilineage differentiation potential of the Nes-GFP ${ }^{+}$SLCs have not yet been reported for SLCs in the literature $[9,10]$. These differentiation properties were very similar to the characteristics of the neural crest stem cells (NCSCs), which have multilineage differentiation capacity and have been derived from bone marrow, dorsal root ganglia, skin, and oral mucosa lamina propria [36-39]. A number of previous studies also hypothesized that many properties of LCs and their progenitors were compatible with a neural crest origin based on the high levels of Nestin expression in adult NCSCs $[14,15,40,41]$. To address this issue, we detected the expression of the neural crest marker $\mathrm{p} 75$ and found that it was co-expressed with Nestin in both the interstitial testis and in cultured Nes-GFP ${ }^{+}$spheres (Supplementary information, Figure S9), providing evidence of the developmental origin of LCs. Genetic lineage tracing (Crelox recombination) of the neural crest derivatives would provide more information on the origin of LCs.

Testosterone is essential for spermatogenesis, fertility, and the maintenance of the male phenotype. To date, testosterone replacement therapy has not proven to be fully satisfactory for male hypogonadal patients as the available testosterone formulations are unable to reproduce the physiological patterns of testosterone secretion [42]. As an alternative, SLCs have the potential to reverse the age-related declines in testosterone levels in aging men and to stimulate reproductive function in hypogonadal males as a physiological means of androgen replacement $[10,16]$. The putative SLCs should have the ability to colonize the interstitium and subsequently differentiate in vivo. Our experiments showed that transplanted Nes$\mathrm{GFP}^{+}$cells colonized the testicular interstitium, differentiated into LCs, partially rescued testosterone production, and facilitated meiotic and early post-meiotic spermatogenic recovery. These observations were further confirmed by the mouse senescence model. In the rat model, although the transplantation of Nes-GFP ${ }^{+}$cells partially recovered the testosterone production, the serum testosterone levels still remained relatively low compared with the normal group (Supplementary information, Figure S5). We speculate that it might be due to the species difference. We injected the mouse PKH26-labeled Nes$\mathrm{GFP}^{+}$cells into EDS-treated rat testis; although the transplanted cells colonized the interstitial area, the rat testis might not be the optimal microenvironment for mouse SLCs. It is worthy to note that EDS treatment does not deplete adult mouse LCs, it is possible that the transplanted Nes-GFP ${ }^{+}$cells support or enhance the function of the "existing endogenous" LC population, indirectly increasing testosterone production. Because one of the mechanisms for adult stem cell therapy is paracrine-mediated action for the recovery of the tissue injury [43-45], whether or not transplanted $\mathrm{Nes}^{+}$SLCs could enhance recovery of endogenous LCs needs to be elucidated in the future studies.

Because Nestin is an intracellular intermediate filament protein, it is difficult to use it as a marker to prospectively isolate SLCs in non-transgenic animal or human samples. It is important to find surface markers specific for $\mathrm{Nes}^{+}$cells. Here we identified that $\mathrm{Nes}-\mathrm{GFP}^{+}$ cells highly expressed CD51 $(93.53 \% \pm 3.10 \%)$ and found that the $\mathrm{CD} 51^{+}$cells could continuously proliferate and exhibited self-renewal and multilineage differentiation ability. More importantly, similar to Nes-GFP ${ }^{+}$SLCs, $\mathrm{CD} 51^{+}$cells could differentiate into mature LCs and produce testosterone in vitro. These results suggest that CD51 might be a specific cell surface marker for SLCs. In addition, p75 was also highly expressed by testis Nes$\mathrm{GFP}^{+}$cells $(74.73 \% \pm 1.89 \%)$. Whether it can serve as another marker for SLCs needs to be further studied.

In conclusion, we have identified nestin as a potential marker of SLCs. Additionally, Nes-GFP ${ }^{+}$cells can be efficiently enriched from neonatal testes and exhibit self-renewal and multilineage differentiation potential. Moreover, transplanted Nes-GFP ${ }^{+}$cells appeared to colonize the host interstitium and differentiated into functional testosterone-producing LCs to partially rescue spermatogenesis. Thus, this study provides groundwork for SLC isolation, which would shed new light on the development of cell replacement therapies for testosterone deficiency or decline in male patients.

\section{Materials and Methods}

\section{Animals \\ The homozygous transgenic mice that express enhanced GFP under the control of the Nestin promoter (Nes-GFP) in the C57BL/6 genetic background were provided by Dr Masahiro Ya- maguchi [46]. C57BL/6 mice and Sprague-Dawley rats were pur- chased from the Animal Center of Sun Yat-sen University (Guang-}


zhou, China). All animal studies were carried out in accordance with the guidelines of the Sun Yat-sen University Institutional Animal Care and Use Committee.

\section{Isolation and culture of Nestin-positive cells from Nes-GFP} transgenic mice

The testes were dissected from 7-day-old Nes-GFP or C57BL/6 mice, and the tunica albuginea was carefully removed from each testis and minced into small pieces. The interstitial cells were then dissociated from the seminiferous tubules by digestion with $1 \mathrm{mg} / \mathrm{ml}$ collagenase type IV (Invitrogen) in Dulbecco's modified Eagle's medium (DMEM)/F12 (Invitrogen) at $37{ }^{\circ} \mathrm{C}$ for $15 \mathrm{~min}$. After adding DMEM containing 5\% fetal bovine serum (FBS, Hyclone) to stop the collagenase activity, the samples were centrifuged at $1500 \mathrm{rpm}$ for $5 \mathrm{~min}$ at room temperature; the pellets were washed twice with phosphate-buffered saline (PBS), resuspended in PBS, and filtered through a $45 \mu \mathrm{m}$ filter, thereby excluding seminiferous tubules from the preparation. The suspension was passed through a $20 \mu \mathrm{m}$ strainer, which resulted in single cells. GFP-expressing cells that exhibited fluorescence intensities $\sim 10$ fold greater than the autofluorescence of cells from C57BL/6L mice were enriched by FACS using an Influx Cell Sorter (Becton Dickinson) and were cultured in ultra-low adherent dishes (Corning). The expansion medium adapted for SLC culture [9] was previously published and was used with modifications; this medium consisted of DMEM/F12 (1:1; Gibco) supplemented with $1 \mathrm{nM}$ dexamethasone (Sigma), $1 \mathrm{ng} / \mathrm{ml}$ LIF (Millipore), $5 \mu \mathrm{g} / \mathrm{L}$ insulin-transferrin-sodium selenite (ITS, Sigma), 5\% chicken embryo extract (US Biologicals), $0.1 \mathrm{mM}$-mercaptoethanol (Invitrogen), $1 \%$ non-essential amino acids (Hyclone), 1\% N2 and 2\% B27 supplements (Invitrogen), $20 \mathrm{ng} / \mathrm{ml}$ basic fibroblast growth factor (Invitrogen), epidermal growth factor (PeproTech), platelet-derived growth factor (PeproTech), and oncostatin M (PeproTech). The cultures were kept at $37{ }^{\circ} \mathrm{C}$ in a humidified $5 \% \mathrm{CO}_{2}$ water-jacketed incubator. The medium was changed every 3 days.

\section{$R N A$ isolation and quantitative real-time and reverse tran- scription PCR}

Total RNA from the testes or cells was extracted using an RNeasy mini kit (Qiagen) according to the manufacturer's protocol. Reverse transcription reactions were performed using murine leukemia virus reverse transcriptase and oligo-dT primers (Fermentas). Conventional PCR was performed using LC Taq (Fermentas). Real-time PCR was performed using the Thunderbird SYBR qPCR Mix (Toyobo) according to the manufacturer's instructions. Signals were detected using a Light Cycler 480 Detection System (Roche). The primer sequences are listed in Supplementary information, Table S1.

\section{Flow cytometry}

Primary testicular cells derived from postnatal day 7 Nes-GFP or C57BL/6 mice were used for flow cytometry analysis. For the identification of surface markers, the cells were incubated for 20 min with flow cytometry buffer (1\% BSA in PBS) on ice and were subsequently incubated for $30 \mathrm{~min}$ with primary and secondary antibodies in the dark on ice at $4{ }^{\circ} \mathrm{C}$. Each analysis was performed on at least three separate cell preparations. The primary and secondary antibodies are listed in Supplementary information, Table $\mathrm{S} 2$.

\section{Clonal sphere formation assay}

Dissociated single-cell suspensions of $\mathrm{Nes}-\mathrm{GFP}^{+}$cells were diluted to a density of 500 cells $/ \mathrm{ml}$, after which $2 \mu \mathrm{l} /$ well of the diluted cell suspension was plated in ultra-low attachment 96-well plates (Corning); $150 \mu \mathrm{l}$ of expansion medium was added to each well. Wells containing only one cell were marked and observed every day. Spheres were defined as free-floating spherical structures with a diameter $>50 \mu \mathrm{m}$. Subsequently, primary spheres (day 10) were dissociated into single cells and replated under the same culture conditions as those used for the growth of primary spheres to generate secondary spheres. All spheres in a given well were counted, and the results were expressed as a percentage of the plated cells.

\section{Cell proliferation assay}

Cells were seeded into 96-well plates at a density of 2500 cells/well. Proliferation was examined using CCK8 kits (Dojindo Laboratory) according to the manufacturer's instructions. The absorbance at $450 \mathrm{~nm}$ was measured with a reference wavelength of $630 \mathrm{~nm}$. All assays were repeated eight times. A calibration curve created from average values obtained from six different known cell densities was used to evaluate the proliferation ability of the Nes$\mathrm{GFP}^{+}$cells.

\section{In vitro differentiation of $\mathrm{Nes}-\mathrm{GFP}^{+}$cells}

Osteogenic differentiation Nes-GFP ${ }^{+}$cells were cultured in $\alpha$-MEM (Invitrogen) containing 20\% FBS, $100 \mu \mathrm{g} / \mathrm{ml}$ ascorbic acid (Sigma), $100 \mathrm{nM}$ dexamethasone (Sigma), $10 \mathrm{mM} \beta$-glycerophosphate (Sigma), and $100 \mathrm{IU} / \mathrm{ml}$ penicillin/streptomycin (Invitrogen). The cells were fed every third day and maintained in culture for 4 weeks. The osteogenic-differentiated cells were fixed and stained with Alizarin red $\mathrm{S}$ to detect the presence of calcium, as previously described [47].

Adipogenic differentiation Adipogenic differentiation was induced by culturing cells in high-glucose DMEM supplemented with $100 \mathrm{nM}$ dexamethasone (Sigma), $10 \mu \mathrm{g} / \mathrm{ml}$ insulin (Sigma), 0.2 $\mathrm{mM}$ indomethacin (Sigma), $0.5 \mathrm{mM}$ 3-isobutyl-1-methylxanthine (Sigma), 10\% FBS and $100 \mathrm{IU} / \mathrm{ml}$ penicillin-streptomycin. The cells were maintained in culture for 4 weeks and were fed every third day. The adipogenic-differentiated cells were confirmed by Oil red O staining, as described previously [47].

Chondrogenic differentiation Chondrogenic differentiation was induced using a cell pellet culture system as previously described [48]. In brief, the Nes-GFP ${ }^{+}$cells were suspended in a $15 \mathrm{ml}$ conical tube containing $2 \mathrm{ml}$ of induction medium consisting of DMEM (Invitrogen) with 3\% FBS, $10 \mathrm{ng} / \mathrm{ml}$ tumor growth factor (TGF)- $\beta 3$ (PeproTech), $1 \times$ ITS (Sigma), and $1 \mathrm{mM}$ pyruvate (Sigma). The cells were fed every third day for 4 weeks, and the chondrocytes were identified by toluidine blue (Sigma) staining, as described previously [49].

Neurogenic differentiation Neural differentiation of the Nes$\mathrm{GFP}^{+}$cells was induced by plating cells onto poly-D-lysine/ laminin-coated 24-well plates in N2B27 medium containing 10 $\mathrm{ng} / \mathrm{ml}$ brain-derived neurotrophic factor and $10 \mathrm{ng} / \mathrm{ml}$ neurotrophin-3 (PeproTech), and the cells were maintained for 2 weeks. For astroglial differentiation, the Nes- $\mathrm{GFP}^{+}$cells were exposed to 
$1 \%$ FBS and bone morphogenic protein (BMP)-4 (10 ng/ml; PeproTech) in N2B27 medium for 7 days [50]. At each experimental endpoint, the differentiated cells were identified by immunostaining using the Tuj-1 and GFAP antibodies shown in Supplementary information, Table S2, or total RNA was extracted for RT-PCR analysis.

LC differentiation For LC lineage differentiation, the Nes-GFP ${ }^{+}$ cells were replated in fresh differentiation-inducing medium containing phenol red-free DMEM/F12, 2\% FCS, 10 ng/ml PDGFBB (PeproTech), 1 ng/ml LH (PeproTech), 1 nM thyroid hormone (PeproTech), $70 \mathrm{ng} / \mathrm{ml}$ insulin-like growth factor 1 (IGF1, PeproTech), and ITS supplement (Sigma), and they were incubated for 7 days, as previously described [9]. Differentiation was subsequently confirmed by RT-PCR and immunostaining for LC lineage markers (antibodies shown in Supplementary information, Table S2).

\section{Testosterone concentration assay}

The cell culture supernatants and sera were collected at each experimental time point for the quantitative determination of testosterone. Testosterone levels were measured using a commercially available ELISA kit (R\&D Systems, Minneapolis, MN, USA), according to the manufacturer's instructions.

\section{Nes-GFP ${ }^{+}$cell transplantation}

For evaluating whether $\mathrm{Nes}-\mathrm{GFP}^{+}$cell injection could facilitate the recovery of LC dysfunction, the $\mathrm{Nes}-\mathrm{GFP}^{+}$cells were washed with PBS and stained with the red fluorescent dye PKH26 (Sigma) according to the manufacturer's instructions. Sixty 3-monthold, twelve 22-month-old male C57BL/6 mice, and eighteen rats ( $n=6$ for each group at each time point) were used in this study. EDS was provided by Dr Renshan Ge (Wenzhou Medical College) and was injected intraperitoneally with either a single dose of 160 (mice) or 75 (rats) $\mathrm{mg} / \mathrm{kg}$ body weight. This treatment resulted in the elimination of LCs in the adult testis within 4 days of treatment in rats [51]. Approximately $1 \times 10^{6} \mathrm{PKH} 26$-labeled Nes-GFP ${ }^{+}$cells in $20 \mu \mathrm{l}$ of PBS were injected into the parenchyma of the recipient testes 4 days after the 3-month-old mice or rats had received EDS or into the parenchyma of the testes in 22-month-old mice. The control animals for the experimental group were EDS-treated mice or rats and 3-month- or 22-month-old normal mice that received a testicular injection of the same volume of saline. Testes and sera from all animals were tested 0, 4, 7, 10, 12, and 14 days after EDS treatment and were examined by qRT-PCR or histological analysis and testosterone concentration measurements.

\section{Tissue and cell preparations}

Nes-GFP mice (postnatal days $0,7,14,28$, and 90) and EDS-treated or cell transplanted 3- and 22-month-old C57BL/6 mice or rats were anesthetized with ketamine $(150 \mu \mathrm{g} / \mathrm{g})$ and xylazine (30 $\mu \mathrm{g} / \mathrm{g}$, Sigma) and then testes were dissected. For immunofluorescence staining, testes fixed with $4 \%$ paraformaldehyde (PFA) in PBS were cryo-embedded in optimal cutting temperature medium (Sakura Finetek). For H\&E histological staining, testes were fixed by immersion in Bouin's fixative and embedded in paraffin. Nes-GFP ${ }^{+}$spheres were collected at $\mathrm{P}_{6}$ and fixed in 4\% PFA for 20 min at room temperature. The cell spheres were subsequently embedded in OCT medium (Sakura Finetek) for frozen sections. Testis tissue and cell spheres were sectioned at a thickness of $5 \mu \mathrm{m}$.

\section{Histological analysis}

At specific time points, sections were boiled in 0.01 Mcitrate $(\mathrm{pH}$ 6.0 ) or cells were blocked by incubation in 5\% normal serum and $0.1 \%$ Triton $\mathrm{X}-100$ (Hyclone) in PBS for $1 \mathrm{~h}$ at room temperature, followed by incubation with primary antibodies at $4{ }^{\circ} \mathrm{C}$ overnight. The sections were then incubated with secondary antibodies at room temperature for $1 \mathrm{~h}$. The primary and secondary antibodies used are listed in Supplementary information, Table S2. Negative controls were prepared by substituting PBS for the primary antibody. All images were obtained using an LSM710 confocal microscope (Zeiss) and were analyzed using the Image J software. For quantitative cell analyses, the GFP-, PH3-, SYCP1- and SY$\mathrm{CP} 3$-positive cells were counted in sections at $400 \times$ magnification in the Nes-GFP mice (postnatal days 0, 7, 14, 28, and 90) or the C57/BL6 mice (EDS-treated or cell-transplanted 3-, and 22-month old mice) and rats, respectively. Three sections per slide and three slides prepared from the upper-, lower-, and mid-portions of the testes per animal were counted in different groups. In inside of seminiferous tubules of the testis, the percentages of PH3-, SYCP1-, SYCP3-positive cells were counted and divided by the total number of cells which excluded elongated spermatids and sperm, respectively [52]. The percentage of $\mathrm{Nes}-\mathrm{GFP}^{+}$cells was calculated to the number of total cells in testis interstitum.

\section{Statistics}

All data are presented as the mean \pm SEM obtained from at least three independent experiments. Comparisons between the groups were performed using a one-way analysis of variance (ANOVA) with Newman-Keuls post hoc comparison. $P<0.05$ was considered statistically significant.

\section{Acknowledgments}

We thank Dr Renshan Ge for providing EDS. This study was supported by the National Basic Research Program of China (2010CB945400 and 2012CBA01302), the National Natural Science Foundation of China (31171398, 81200945, 81270696, and 81302223), the Key Scientific and Technological Projects of Guangdong Province (2007A032100003), the Key Scientific and Technological Program of Guangzhou City (201400000003-3, 201300000089, and 201300000094), the Guangdong Natural Science Foundation (S2011040002505), Guangdong Province Universities and Colleges Pearl River Scholar Funded Scheme (GDUPS, 2013), and Guangdong Department of Science \& Technology Translational Medicine Center grant (2011A080300002).

\section{References}

1 Odell WD, Swerdloff RS, Bain J, Wollesen F, Grover PK. The effect of sexual maturation on testicular response to $\mathrm{LH}$ stimulation of testosterone secretion in the intact rat. Endocrinology 1974; 95:1380-1384.

2 Haider SG. Cell biology of Leydig cells in the testis. Int Rev Cytol 2004; 233:181-241.

3 McHenry Martin C. Testosterone deficiency in older men: a problem worth treating. Consult Pharm 2012; 27:152-163.

4 Huhtaniemi I. Late-onset hypogonadism: current concepts and controversies of pathogenesis, diagnosis and treatment. Asian J Androl 2014; 16:192-202. 
5 Machluf M, Orsola A, Boorjian S, Kershen R, Atala A. Microencapsulation of Leydig cells: a system for testosterone supplementation. Endocrinology 2003; 144:4975-4979.

6 Mendis-Handagama SM, Ariyaratne HB. Differentiation of the adult Leydig cell population in the postnatal testis. Biol Reprod 2001; 65:660-671.

7 Keeney DS, Mendis-Handagama SM, Zirkin BR, Ewing LL. Effect of long term deprivation of luteinizing hormone on Leydig cell volume, Leydig cell number, and steroidogenic capacity of the rat testis. Endocrinology 1988; 123:29062915.

8 Lo KC, Lei Z, Rao ChV, Beck J, Lamb DJ. De novo testosterone production in luteinizing hormone receptor knockout mice after transplantation of leydig stem cells. Endocrinology 2004; 145:4011-4015.

9 Ge RS, Dong Q, Sottas CM, Papadopoulos V, Zirkin BR, Hardy MP. In search of rat stem Leydig cells: identification, isolation, and lineage-specific development. Proc Natl Acad Sci USA 2006; 103:2719-2724.

10 Stanley E, Lin CY, Jin S, et al. Identification, proliferation, and differentiation of adult Leydig stem cells. Endocrinology 2012; 153:5002-5010.

11 Wiese C, Rolletschek A, Kania G, et al. Nestin expression a property of multi-lineage progenitor cells? Cell Mol Life Sci 2004; 61:2510-2522.

12 Mendez-Ferrer S, Michurina TV, Ferraro F, et al. Mesenchymal and haematopoietic stem cells form a unique bone marrow niche. Nature 2010; 466:829-834.

13 Li L, Mignone J, Yang M, et al. Nestin expression in hair follicle sheath progenitor cells. Proc Natl Acad Sci USA 2003; 100:9958-9961.

14 Davidoff MS, Middendorff R, Enikolopov G, Riethmacher D, Holstein AF, Muller D. Progenitor cells of the testosterone-producing Leydig cells revealed. J Cell Biol 2004; 167:935-944.

15 Lobo MV, Arenas MI, Alonso FJ, et al. Nestin, a neuroectodermal stem cell marker molecule, is expressed in Leydig cells of the human testis and in some specific cell types from human testicular tumours. Cell Tissue Res 2004; 316:369-376.

16 Ge RS, Dong Q, Sottas CM, Chen H, Zirkin BR, Hardy MP. Gene expression in rat leydig cells during development from the progenitor to adult stage: a cluster analysis. Biol Reprod 2005; 72:1405-1415.

17 Yoshinaga K, Nishikawa S, Ogawa M, et al. Role of c-kit in mouse spermatogenesis: identification of spermatogonia as a specific site of c-kit expression and function. Development 1991; 113:689-699.

18 Ketola I, Pentikainen V, Vaskivuo T, et al. Expression of transcription factor GATA-4 during human testicular development and disease. J Clin Endocrinol Metab 2000; 85:3925-3931.

19 Ketola I, Rahman N, Toppari J, et al. Expression and regulation of transcription factors GATA-4 and GATA-6 in developing mouse testis. Endocrinology 1999; 140:1470-1480.

20 Sawamoto K, Nakao N, Kakishita K, et al. Generation of dopaminergic neurons in the adult brain from mesencephalic precursor cells labeled with a nestin-GFP transgene. J Neurosci 2001; 21:3895-3903.

21 Blanpain C, Lowry WE, Geoghegan A, Polak L, Fuchs E. Self-renewal, multipotency, and the existence of two cell populations within an epithelial stem cell niche. Cell 2004; 118:635-648.

22 Parker KL, Rice DA, Lala DS, et al. Steroidogenic factor 1: an essential mediator of endocrine development. Recent Prog Horm Res 2002; 57:19-36.

23 Mignone JL, Kukekov V, Chiang AS, Steindler D, Enikolopov G. Neural stem and progenitor cells in nestin-GFP transgenic mice. J Comp Neurol 2004; 469:311-324.

24 Chen H, Liu J, Luo L, Baig MU, Kim JM, Zirkin BR. Vitamin E, aging and Leydig cell steroidogenesis. Exp Gerontol 2005; 40:728-736.

25 Midzak AS, Chen H, Papadopoulos V, Zirkin BR. Leydig cell aging and the mechanisms of reduced testosterone synthesis. Mol Cell Endocrinol 2009; 299:23-31.

26 Chen H, Hardy MP, Zirkin BR. Age-related decreases in Leydig cell testosterone production are not restored by exposure to LH in vitro. Endocrinology 2002; 143:1637-1642.

27 Andersson AM, Jorgensen N, Frydelund-Larsen L, Rajpert-De Meyts E, Skakkebaek NE. Impaired Leydig cell function in infertile men: a study of 357 idiopathic infertile men and 318 proven fertile controls. J Clin Endocrinol Metab 2004; 89:3161-3167.

28 Ruwanpura SM, McLachlan RI, Meachem SJ. Hormonal regulation of male germ cell development. J Endocrinol 2010; 205:117-131.

29 McLachlan RI, O’Donnell L, Meachem SJ, et al. Identification of specific sites of hormonal regulation in spermatogenesis in rats, monkeys, and man. Recent Prog Horm Res 2002; 57:149-179.

30 Haywood M, Spaliviero J, Jimemez M, King NJ, Handelsman DJ, Allan CM. Sertoli and germ cell development in hypogonadal (hpg) mice expressing transgenic follicle-stimulating hormone alone or in combination with testosterone. Endocrinology 2003; 144:509-517.

31 La Salle S, Sun F, Handel MA. Isolation and short-term culture of mouse spermatocytes for analysis of meiosis. Methods Mol Biol 2009; 558:279-297.

32 Sato T, Katagiri K, Gohbara A, et al. In vitro production of functional sperm in cultured neonatal mouse testes. Nature 2011; 471:504-507.

33 Ozerdem U, Grako KA, Dahlin-Huppe K, Monosov E, Stallcup WB. NG2 proteoglycan is expressed exclusively by mural cells during vascular morphogenesis. Dev Dyn 2001; 222:218227.

34 Li Q, Yu Y, Bischoff J, Mulliken JB, Olsen BR. Differential expression of CD146 in tissues and endothelial cells derived from infantile haemangioma and normal human skin. J Pathol 2003; 201:296-302.

35 Park D, Xiang AP, Mao FF, et al. Nestin is required for the proper self-renewal of neural stem cells. Stem Cells 2010; 28:2162-2171.

36 Achilleos A, Trainor PA. Neural crest stem cells: discovery, properties and potential for therapy. Cell Res 2012; 22:288304.

37 Davies LC, Locke M, Webb RD, et al. A multipotent neural crest-derived progenitor cell population is resident within the oral mucosa lamina propria. Stem Cells Dev 2010; 19:819830 .

38 Kaltschmidt B, Kaltschmidt C, Widera D. Adult craniofacial 
stem cells: sources and relation to the neural crest. Stem Cell Rev 2012; 8:658-671.

39 Pierret C, Spears K, Maruniak JA, Kirk MD. Neural crest as the source of adult stem cells. Stem Cells Dev 2006; 15:286291.

40 Davidoff MS, Schulze W, Middendorff R, Holstein AF. The Leydig cell of the human testis - a new member of the diffuse neuroendocrine system. Cell Tissue Res 1993; 271:429439.

41 Davidoff MS, Middendorff R, Kofuncu E, Muller D, Jezek D, Holstein AF. Leydig cells of the human testis possess astrocyte and oligodendrocyte marker molecules. Acta Histochem 2002; 104:39-49.

42 Giagulli VA, Triggiani V, Corona G, et al. Evidence-based medicine update on testosterone replacement therapy (TRT) in male hypogonadism: focus on new formulations. Curr Pharm Des 2011; 17:1500-1511.

43 Gharaibeh B, Lavasani M, Cummins JH, Huard J. Terminal differentiation is not a major determinant for the success of stem cell therapy - cross-talk between muscle-derived stem cells and host cells. Stem Cell Res Ther 2011; 2:31.

44 Maumus M, Guerit D, Toupet K, Jorgensen C, Noel D. Mesenchymal stem cell-based therapies in regenerative medicine: applications in rheumatology. Stem Cell Res Ther 2011; 2:14.

45 Gnecchi M, He H, Liang OD, et al. Paracrine action accounts for marked protection of ischemic heart by Akt-modified mesenchymal stem cells. Nat Med 2005; 11:367-368.

46 Yamaguchi M, Saito H, Suzuki M, Mori K. Visualization of neurogenesis in the central nervous system using nestin promoter-GFP transgenic mice. Neuroreport 2000; 11:1991-
1996.

47 Salero E, Blenkinsop TA, Corneo B, et al. Adult human RPE can be activated into a multipotent stem cell that produces mesenchymal derivatives. Cell Stem Cell 2012; 10:88-95.

$48 \mathrm{Ke} \mathrm{H}$, Wang P, Yu W, et al. Derivation, characterization and gene modification of cynomolgus monkey mesenchymal stem cells. Differentiation 2009; 77:256-262.

49 Pinho S, Lacombe J, Hanoun M, et al. PDGFRalpha and CD51 mark human nestin+ sphere-forming mesenchymal stem cells capable of hematopoietic progenitor cell expansion. $J$ Exp Med 2013; 210:1351-1367.

50 Sheng C, Zheng Q, Wu J, et al. Direct reprogramming of Sertoli cells into multipotent neural stem cells by defined factors. Cell Res 2012; 22:208-218.

51 Teerds KJ, De Rooij DG, Rommerts FF, Wensing CJ. The regulation of the proliferation and differentiation of rat Leydig cell precursor cells after EDS administration or daily HCG treatment. J Androl 1988; 9:343-351.

52 Huckins C, Oakberg EF. Morphological and quantitative analysis of spermatogonia in mouse testes using whole mounted seminiferous tubules. II. The irradiated testes. Anat Rec 1978, 192:529-542.

(Supplementary information is linked to the online version of the paper on the Cell Research website.)

(c) (i) (3) $€$ This work is licensed under the Creative Commons Attribution-NonCommercial-No Derivative Works 3.0 Unported License. To view a copy of this license, visit http:// creativecommons.org/licenses/by-nc-nd/3.0 